\title{
FREDHOLM EQUATIONS ON A HILBERT SPACE OF ANALYTIC FUNCTIONS
}

\author{
BY \\ CLASINE VAN WINTER( $\left.{ }^{(}\right)$
}

\begin{abstract}
It is shown that the Hardy class $\mathscr{H}^{2}$ for the upper half-plane is equal to the set of functions $f[r \exp (i \phi)]$ which are analytic in the open half-plane and squareintegrable with respect to $r$ for $0<\phi<\pi$. A function $f$ is in $\mathfrak{S}^{2}$ if and only if its Mellin transform with respect to $r$ is a constant times $f(t) \exp (\phi t-i \phi / 2)$, where $f$ must belong to a certain $\mathfrak{L}^{2}$-space. This result enables $f$ in $\mathfrak{S}^{2}$ to be constructed from its boundary values on the positive real axis.

A study is made of a class $\mathfrak{R}$ consisting of integral operators $K$ on $\mathscr{S}^{2}$ having kernels $K\left(r, r^{\prime}, \phi\right)$ which are square-integrable with respect to $r$ and $r^{\prime}$. It is found that $\mathfrak{R}$ is a Hilbert space and is a proper subset of the Schmidt class. The class $\mathfrak{R}$ is not an ideal in the algebra $\mathfrak{B}$ of all bounded operators on $\mathfrak{S}^{2}$, but there is a Banach algebra $\mathfrak{A}$ which is dense in $\mathfrak{B}$ and contains $\mathfrak{A}$ as an ideal. An operator $A$ in $\mathfrak{A}$ is associated with a family of operators $A(\phi)$ on $\mathfrak{L}^{2}[0, \infty)$. As a result, a Fredholm equation on $\mathfrak{S}^{2}$ with a kernel $K$ in $\mathfrak{\Omega}$ is associated with a family of equations on $\mathfrak{L}^{2}[0, \infty)$ with kernels $K(\phi)$. The solution of the equation with kernel $K(\phi)$ has an analytic continuation which solves the equation on $\mathfrak{S}^{2}$ with kernel $K$, and all solutions in $\mathfrak{S}^{2}$ can be obtained in this way.

Arguments based on the Mellin transform show that the kernels $K\left(r, r^{\prime}, \phi\right)$ of operators in $\mathfrak{N}$ form a Hardy class $\mathfrak{S}^{2}(2)$ of functions of two variables, one complex and one real. A generalization leads to Hardy classes $\mathfrak{S}^{2}(n)$ of functions of $n$ variables. On $\mathfrak{S}^{2}(n)$, there is a class of operators $\mathfrak{I}(n)$ whose kernels form a class $\mathfrak{S}^{2}(2 n)$.

This formalism was developed with a view to the $n$-body problem in quantum mechanics. It is explained that the results on $\mathfrak{S}^{2}(n-1)$ are instrumental in evaluating quantities which occur in the theory of $n$-particle scattering.
\end{abstract}

\section{Introduction.}

1.1. Motivation. Consider an integral equation of the form

$$
f(r)=g(r)+\int_{0}^{\infty}(r-\lambda)^{-1} V\left(r, r^{\prime}\right) f\left(r^{\prime}\right) d r^{\prime} \quad(0 \leqq r<\infty),
$$

where $f$ and $g$ are in $\mathfrak{L}^{2}$, the function $g$ is known and $f$ is to be found. Let $\lambda$ be a complex number and suppose that $\int_{0}^{\infty}\left|V\left(r, r^{\prime}\right)\right|^{2} d r^{\prime}$ exists and is bounded uniformly

Presented to the Society, January 25, 1970 under the title A Banach algebra of integral operators on the Hardy space $H^{2}$; received by the editors July 8, 1970 and, in revised form, February 2, 1971.

AMS 1969 subject classifications. Primary 3067, 3085, 4511, 4615, 4630, 4725, 4770; Secondary 4430, 4665, 7045, 8130, 8145, 8146, 8147.

Key words and phrases. Fredholm equation, Hilbert space of analytic functions, Hardy class, Schmidt class, integral operator, operator algebra, operator ideal, Mellin transform, Paley-Wiener theorem, $\boldsymbol{n}$-body problem in quantum mechanics, scattering theory.

( ${ }^{1}$ This work was supported in part by the National Science Foundation under grant GP-13476. 
in $r$. This gives an equation which occurs in quantum mechanics when one studies the scattering of two particles [3, p. 1077], [4, pp. 178-197], [7, pp. 319-322]. The variable $r$ would represent the kinetic energy of the relative motion of the particles, $V$ describing their interaction. The resolvent of the two-particle Hamilton operator satisfies an equation like equation (1.1).

If $\lambda$ is not real and $V$ satisfies the assumption stated above, the equation has a kernel in the Schmidt class. Hence it can be solved in terms of a quotient of two Fredholm series. If $\lambda$ tends to the positive real axis, however, the Fredholm series cease to converge and so the solution breaks down. This difficulty is directly related to the Hamilton operator having a continuous spectrum along the positive real axis. Now, scattering theory can be regarded as the physics approach to studying a continuous spectrum. In fact, for applications to physics it is essential that we try and tackle equation (1.1) in the case that $\lambda$ takes positive real values.

At this point, it is helpful to observe that one does not really need a complete solution of equation (1.1). It can be shown that observable scattering quantities have the general structure of

$$
\int_{0}^{\infty} f(r) h(r) d r
$$

where $f$ satisfies equation (1.1) and $h$ is a suitable function in $\mathfrak{Q}^{2}$. Through equation (1.1), $f$ will depend on $\lambda$, hence $f=f(r, \lambda)$. Further simplifications arise from the fact that one merely requires

$$
\lim _{\varepsilon \rightarrow 0} \int_{0}^{\infty} f(r, l-i \varepsilon) h(r) d r
$$

it being understood that $l \geqq 0$ and $\varepsilon \geqq 0$. Thirdly, it often suffices to consider functions $f$ corresponding to smooth functions $g$ in equation (1.1), and it may often be assumed that $V$ also has suitable smoothness properties. In this paper, we develop an approach to equation (1.1) and related equations which exploits all three simplifying circumstances.

1.2. The Hilbert space $\mathfrak{S}^{2}$. To begin with, we only consider functions $g$ which are boundary values, as $\phi$ tends to 0 , of functions $g\left(r e^{i \phi}\right)$ which are analytic in the open upper half-plane and have the property that

$$
\int_{0}^{\infty}\left|g\left(r e^{i \phi}\right)\right|^{2} d r
$$

exists and is bounded, uniformly in $\phi$ for $0<\phi<\pi$. For applications to physics, it is actually sufficient to assume that $g$ is analytic and that the integral (1.4) converges merely in some sector $0<\phi<\psi$, with $\psi<\pi$, but in the present paper we prefer the notational convenience which results from working in the whole upper half-plane.

According to Theorem 2.7 below, the set of functions $g$ considered in the previous paragraph is precisely the Hardy class $\mathfrak{S}^{2}$ for the upper half-plane. This is usually 
studied by considering analytic functions $g(x+i y)$ and letting $y$ vary. In the present paper, however, it is essential that we use polar coordinates $r, \phi$ and vary $\phi$. This results in an approach to $\mathfrak{S}^{2}$ which appears to be new. Our major analytical tool is the Mellin transform. It is shown in Theorem 2.9 that a function $g\left(r e^{i \phi}\right)$ is in $\mathfrak{S}^{2}$ if and only if its Mellin transform with respect to $r$ is of the form $g(t) \exp \left(\phi t-\frac{1}{2} i \phi\right)$, where $g$ must satisfy

$$
\int_{-\infty}^{\infty}\left(1+e^{2 \pi t}\right)|g(t)|^{2} d t<\infty
$$

This result can be regarded as an analogue of the Paley-Wiener theorem [2, p. 131], [5, p. 8], [9, p. 128]. It enables $g$ in $\mathfrak{S}^{2}$ to be constructed explicitly from its boundary values $g(r)$ on the half-line $r \geqq 0$ and is also instrumental in showing that the set of boundary values $g(r)$ of functions $g$ in $\mathfrak{S}^{2}$ is dense in the space of functions which are defined on $r \geqq 0$ and are square-integrable. The latter space is simply denoted by $\mathfrak{Q}^{2}$ in the present paper. The above facts are discussed in Remark 2.11 and Theorem 2.17.

If $f$ and $h$ are in $\mathfrak{S}^{2}$, then

$$
\int_{0}^{\infty} f\left(r e^{i \phi}\right) h\left(r e^{i \phi}\right) e^{i \phi} d r
$$

does not depend on $\phi$, by Corollary 2.8. Suppose, therefore, that the solution $f(r)$ of equation (1.1) is the boundary value of some $f$ in $\mathfrak{S}^{2}$, and let the same hold true for $h(r)$ in the expression (1.2). Instead of actually finding the quantity (1.2), it then suffices to evaluate the integral (1.6) for some convenient nonzero $\phi$. Now suppose that $V$ has analyticity properties which enable equation (1.1) to be continued analytically, $f\left(r e^{i \phi}\right)$ being a solution of the continued equation. All we have to do, then, is to solve equation (1.1) for some convenient $\phi$ and insert the solution in the integral (1.6). But if $r$ in equation (1.1) is replaced by $r e^{i \phi}$, with $0<\phi \leqq \pi$, then there does not seem to be any problem involved in writing $\lambda=l-i \varepsilon$ and letting $\varepsilon$ tend to 0 . Hence, it should be possible to evaluate the expression (1.3) by first rotating the path of integration, next performing the limit with respect to $\varepsilon$. This idea was put forward by the author in reference [12]. Working out all details also requires the results of references [10] and [11].

1.3. The class of integral operators $\Re$. For the above ideas to be applicable, it is not essential that $\lambda$ enters in the integral kernel precisely in the form (1.1). In the main body of this paper, we therefore drop $\lambda$ altogether and we address ourselves to the general problem of studying a class $\mathfrak{R}$ of integral operators $K$ on $\mathfrak{S}^{2}$ which act according to

$$
K f\left(r e^{i \phi}\right)=\int_{0}^{\infty} K\left(r, r^{\prime}, \phi\right) f\left(r^{\prime} e^{i \phi}\right) e^{i \phi} d r^{\prime},
$$

the integral kernel satisfying

$$
\int_{0}^{\infty} \int_{0}^{\infty}\left|K\left(r, r^{\prime}, \phi\right)\right|^{2} d r d r^{\prime}<\infty
$$


The set of functions $K$ which are kernels of operators $K$ in $\Re$ is also denoted by $\AA$. Theorem 3.5 says that an integral operator $K$ on $\mathfrak{S}^{2}$ is in $\mathfrak{\AA}$ if and only if the Mellin transform of its kernel has certain properties analogous to equation(1.5). According to Theorem 3.8, one can define an inner product so that $\Re$ becomes a Hilbert space. This space is a proper subclass of the Schmidt class $\subseteq$ on $\mathfrak{S}^{2}$, by Theorem 3.11. In the Schmidt norm, $\mathfrak{R}$ is dense in $\mathfrak{S}$, by Theorem 3.12 .

1.4. The class of operators $\mathfrak{A}$. It is well known that the Schmidt class $\mathfrak{S}$ is an ideal in the algebra $\mathfrak{B}$ consisting of all bounded linear operators on $\mathfrak{S}^{2}$. An example in $\S 4.1$ shows that $\mathfrak{R}$ is not an ideal in $\mathfrak{B}$. This may give rise to various difficulties in the applications which we have in mind. $\$ \$ 4.2-4.4$ are therefore devoted to a Banach algebra $\mathfrak{A}$ of operators on $\mathfrak{S}^{2}$ which is a proper subset of $\mathfrak{B}$ and contains $\mathfrak{A}$ as an ideal. The set $\mathfrak{A}$ is dense in $\mathfrak{B}$ in the strong topology, by Theorem 4.19, but it is pointed out in Corollary 4.21 that there are operators in $\mathfrak{B}$ which cannot be approximated uniformly by operators in $\mathfrak{A}$. Any operator of finite rank obviously belongs to $\mathfrak{B}$, but Remark 4.18 shows that there are operators of rank 1 which do not belong to $\mathfrak{A}$.

If $f$ runs through $\mathfrak{S}^{2}$, but $\phi$ is kept fixed, so that $f\left(r e^{i \phi}\right)$ is regarded as a function of $r$ only, then the functions $f\left(r e^{i \phi}\right)$ yield a set $\mathfrak{D}(\phi)$ which is dense in $\mathfrak{Q}^{2}$, by Theorem 2.17. Now let $A$ be in $\mathfrak{A}$. The restriction of $A f\left(r e^{i \phi}\right)$ to fixed $\phi$ can then be written in the form $A(\phi) f\left(r e^{i \phi}\right)$, this defining an operator $A(\phi)$ having a unique continuous extension to all of $\mathfrak{Q}^{2}$, by Lemma 4.9. This is the decisive feature of the class $\mathfrak{A}$ which makes it useful for applications. In particular, it is due to this property that one can consider an equation on $\mathfrak{S}^{2}$ involving operators in $\mathfrak{A}$, restrict the equation to $\phi=0$, and obtain a meaningful equation on $\mathfrak{Q}^{2}$.

Given some fixed $A$ in $\mathfrak{A}$, the logarithm of the $\mathfrak{Q}^{2}$-norm of $A(\phi)$ is a convex function of $\phi$, by Theorem 4.10.

1.5. Fredholm equations. The properties of the class $\mathfrak{A}$ are used, in particular, in $\S 5$, which discusses Fredholm equations on $\mathfrak{S}^{2}$ with kernels in $\mathfrak{R}$. If $1 / \gamma$ is not an eigenvalue of $K$, one can solve the equation

$$
f\left(r e^{i \phi}\right)=g\left(r e^{i \phi}\right)+\gamma \int_{0}^{\infty} K\left(r, r^{\prime}, \phi\right) f\left(r^{\prime} e^{i \phi}\right) e^{i \phi} d r^{\prime}
$$

with Schmidt-class methods on $\mathfrak{S}^{2}$, next $\phi$ can be restricted to some fixed value. Alternatively, $\phi$ is fixed first, the restriction of $K$ to $\mathscr{D}(\phi)$ is extended to the operator $K(\phi)$ on $\mathfrak{Q}^{2}$, and $f$ is found from an equation on $\mathfrak{Q}^{2}$ with kernel $K(\phi)$. Theorems 5.4 and 5.5 state that the two approaches give exactly the same result. Thus, the restriction of the solution is the solution of the restricted equation. If we think primarily in terms of equation (1.1), and if this is the restriction to $\phi=0$ of an equation on $\mathfrak{S C}^{2}$ with a kernel in $\mathfrak{R}$, then we can also say that the solution of the restricted equation has an analytic continuation which solves the analytically continued equation.

In case $1 / \gamma$ is an eigenvalue of the operator $K$ in equation (1.9), it is also an 
eigenvalue of all operators $K(\phi)$. The restriction to fixed $\phi$ of an eigenvector $f\left(r e^{i \phi}\right)$ of $K$ is an eigenvector of $K(\phi)$, and the functions $f\left(r e^{i \phi}\right)$ obtained in this way together account for all eigenvectors of $K(\phi)$, by Theorem 5.8 .

Summarizing, our program for evaluating the integral (1.3) can be carried through whenever $V\left(r, r^{\prime}\right)$ in equation $(1.1)$ is so as to make $(r-l+i \varepsilon)^{-1} V$ the boundary value $K(0)$ of an operator $K$ in $\Re$.

1.6. Functions of several variables. With arguments based on the Mellin transform, it is shown in $\S 6$ that the functions $K\left(r, r^{\prime}, \phi\right)$ in $\Re$ depend only on $r e^{i \phi}$ and $r^{\prime} e^{i \phi}$. Writing

$$
r=\rho \cos \theta, \quad r^{\prime}=\rho \sin \theta \quad(0 \leqq \rho<\infty, 0 \leqq \theta \leqq \pi / 2)
$$

yields functions $k\left(\rho e^{i \phi}, \theta\right)$ such that

$$
\int_{0}^{\pi / 2} d \theta \int_{0}^{\infty}\left|k\left(\rho e^{i \phi}, \theta\right)\right|^{2} \rho d \rho
$$

exists and is bounded, uniformly in $\phi$ for $0<\phi<\pi$. This result resembles the expression (1.4). Indeed, Theorem 6.2 and Corollary 6.4 state that the functions $K$ in $\AA$ form a Hardy space $\mathfrak{S}^{2}(2)$ of functions of $\rho e^{i \phi}$ and $\theta$, the variable $\theta$ being real and $\rho e^{i \phi}$ taking values in the upper half-plane. This space provides an appropriate framework for studying equations like

$$
\begin{aligned}
& f\left(r_{1}, r_{2}\right)=g\left(r_{1}, r_{2}\right) \\
& \quad+\int_{0}^{\infty} \int_{0}^{\infty}\left[\left(r_{1}^{2}+r_{2}^{2}\right)^{1 / 2}-\lambda\right]^{-1} W\left(r_{1}, r_{2}, r_{1}^{\prime}, r_{2}^{\prime} ; \lambda\right) f\left(r_{1}^{\prime}, r_{2}^{\prime}\right) d r_{1}^{\prime} d r_{2}^{\prime}
\end{aligned}
$$

which occur when one considers the scattering of three particles. If in such equations it is demanded that $f$ be the boundary value of a function in $\mathfrak{S}^{2}(2)$, this means, essentially, that $\mathfrak{\Re}$ is not only regarded as a space of two-particle operators, but at the same time as a space of three-particle functions. The class $\mathfrak{A}$ being an ideal in $\mathfrak{A}$ then means that $\mathfrak{A}$ is an algebra of two-particle operators which can also be applied in a meaningful way in three-particle systems.

The results of the present paper can be extended without difficulty to Hardy spaces $\mathfrak{S}^{2}(n)$ of functions of $n$ variables $(n=2,3, \ldots)$, one complex and $n-1$ real. The space $\mathfrak{S}^{2}(n-1)$ is useful for studying systems of $n$ particles along the lines indicated above. Detailed applications to physics will be published in a separate paper.

\section{The Hilbert space.}

2.1. General properties. Let $f$ be an analytic function, regular in the upper halfplane, and assume that there is a constant $H$ such that

$$
\int_{-\infty}^{\infty}|f(x+i y)|^{2} d x<H \quad(y>0)
$$


Then there exists a function $f(x)$ in $\mathfrak{2}^{2}$ such that, as $y$ tends to 0 , the function $f(x+i y)$ tends to $f(x)$ for almost every $x$. Furthermore,

$$
\lim _{y \rightarrow 0} \int_{-\infty}^{\infty}|f(x+i y)-f(x)|^{2} d x=0 .
$$

In the upper half-plane, $f$ is the Poisson integral of its boundary function according to

$$
f(x+i y)=(y / \pi) \int_{-\infty}^{\infty}\left[(u-x)^{2}+y^{2}\right]^{-1} f(u) d u .
$$

These facts are discussed in Titchmarsh's book [9, pp. 125-128].

The set of all functions having the properties listed above is called the Hardy class $\mathfrak{S}^{2}$. With the inner product

$$
(f, g)=\int_{-\infty}^{\infty} f(x) \bar{g}(x) d x,
$$

$\mathfrak{S}^{2}$ becomes a Hilbert space, which we likewise denote by $\mathfrak{S}^{2}$, see Hoffman's book [2, p. 104].

Now consider the function class $\mathbb{S S}$ characterized by the following definition.

Definition 2.1. The class of functions $f$ which are analytic, regular in the upper half-plane, and have the property that

$$
\int_{0}^{\infty}\left|f\left(r e^{i \phi}\right)\right|^{2} d r
$$

exists and is bounded, uniformly in $\phi$ for $0<\phi<\pi$, is denoted by (SS.

It is our first objective to show that a function $f$ is in $\mathbb{S S}$ if and only if it is in $\mathfrak{S}^{2}$. The proof depends on several lemmas.

LEMMA 2.2. If $f$ is in $\mathfrak{S}^{2}$, then it is in 15.

Proof. Since $f$ is in $\mathfrak{S}^{2}$, it can be written as a Poisson integral according to equation (2.3). The integral (2.5) thus takes the form

$$
\begin{aligned}
&(\sin \phi)^{2} \pi^{-2} \int_{0}^{\infty}\left[r^{2} d r \int_{-\infty}^{\infty} \int_{-\infty}^{\infty}|f(u) f(v)|\right. \\
&\left.\times\left(u^{2}+r^{2}-2 u r \cos \phi\right)^{-1}\left(v^{2}+r^{2}-2 v r \cos \phi\right)^{-1} d u d v\right] .
\end{aligned}
$$

It suffices to show that this is bounded uniformly for $0<\phi<\pi$. Writing $u=r \xi$, $v=r \eta$ reduces the integration over $r$ to

$$
\int_{0}^{\infty}|f(r \xi) f(r \eta)| d r \leqq|\xi \eta|^{-1 / 2} \int_{-\infty}^{\infty}|f(u)|^{2} d u .
$$

Thus, it only remains to prove that

$$
\sin \phi \int_{-\infty}^{\infty}|\xi|^{-1 / 2}\left(\xi^{2}+1-2 \xi \cos \phi\right)^{-1} d \xi
$$


is bounded uniformly in $\phi$. On the intervals $-\infty<\xi<-\frac{1}{5}$ and $\frac{1}{5}<\xi<\infty$, the factor $|\xi|^{-1 / 2}$ does not exceed $5^{1 / 2}$. Since

$$
\sin \phi \int_{-\infty}^{\infty}\left(\xi^{2}+1-2 \xi \cos \phi\right)^{-1} d \xi=\pi
$$

the two intervals under discussion do not give difficulties.

Now consider the interval $-\frac{1}{5} \leqq \xi \leqq \frac{1}{5}$ and suppose first that $\sin \phi \leqq \frac{3}{5}$. Then $|\cos \phi| \geqq \frac{4}{5}$, hence $(\xi-\cos \phi)^{2} \geqq \frac{9}{25}$ and

$$
(\sin \phi)\left(\xi^{2}+1-2 \xi \cos \phi\right)^{-1} \leqq \frac{5}{3} .
$$

Next, suppose that $\sin \phi \geqq \frac{3}{5}$. Since $\xi^{2}+1-2 \xi \cos \phi \geqq \sin ^{2} \phi$, this case again gives equation (2.10). Thus, on $-\frac{1}{5} \leqq \xi \leqq \frac{1}{5}$, the integrand in the expression (2.8) does not exceed a constant times $|\xi|^{-1 / 2}$. Since this is an integrable function, we may conclude that the expression (2.6) is bounded uniformly for $0<\phi<\pi$. This completes the proof of the lemma.

2.2. Functions in $\mathfrak{S S}$. In showing that $\mathfrak{S}^{2}$ is contained in $\mathscr{S}$, the essential points are that $f$ in $\mathfrak{S}^{2}$ has boundary values, and that it can be expressed as an integral in terms of these. We proceed to show that functions $f$ in $\mathbb{S S}$ also have these properties. Once this result is available, it is but one step to see that $\mathbb{S}$ is contained in $\mathfrak{S}^{2}$.

LEMMA 2.3. If $f$ is in (S), then there are boundary functions $f(r)$ and $f\left(r e^{i \pi}\right)$ such that

$$
\lim _{\phi \rightarrow \psi} \int_{0}^{\infty}\left|f\left(r e^{i \phi}\right)-f\left(r e^{i \psi}\right)\right|^{2} d r=0 \quad(\psi=0, \pi) .
$$

Proof. The change of variables

$$
r e^{i \phi}=e^{w}, \quad w=u+i v
$$

transforms the upper half-plane into the strip $-\infty<u<\infty, 0<v<\pi$. If $f$ is in $\mathbb{S}$, then the function

$$
F(w)=\left(r e^{i \phi}\right)^{1 / 2} f\left(r e^{i \phi}\right)
$$

is analytic in the strip and has the property that

$$
\int_{-\infty}^{\infty}|F(u+i v)|^{2} d u
$$

exists and is bounded uniformly for $0<v<\pi$. This follows immediately from Definition 2.1 for 85 . From the theory of functions analytic in a strip [5, pp. 3-8], $[9$, p. 131], it follows that there are boundary functions $F(u)$ and $F(u+i \pi)$ such that

$$
\lim _{v \rightarrow \psi} \int_{-\infty}^{\infty}|F(u+i v)-F(u+i \psi)|^{2} d u=0 \quad(\psi=0, \pi) .
$$


Given equation (2.13), the boundary functions $F$ correspond in an obvious way to boundary functions $f$. Expressing equation (2.15) in terms of $f$ and the variable $r e^{i \phi}$ easily gives the desired relation (2.11).

LEMMA 2.4. If $f$ is in 5 and $0<\phi<\pi$, then

$$
\lim _{r \rightarrow 0} r^{1 / 2} f\left(r e^{i \phi}\right)=0, \quad \lim _{r \rightarrow \infty} r^{1 / 2} f\left(r e^{i \phi}\right)=0,
$$

the limits being attained uniformly in $\phi$ in any closed interval $0<\phi_{1} \leqq \phi \leqq \phi_{2}<\pi$.

Proof. In the notation of equation (2.13), it suffices to show that $F(w)$ tends to 0 as $u$ tends to $\pm \infty$, uniformly in $v$ for $0<v_{1} \leqq v \leqq v_{2}<\pi$. Now, $F(w)$ can be written in the form

$$
\begin{aligned}
F(w)= & (2 \pi i)^{-1} \int_{-\infty}^{\infty}(x-w)^{-1} F(x) d x \\
& -(2 \pi i)^{-1} \int_{-\infty}^{\infty}(x+i \pi-w)^{-1} F(x+i \pi) d x
\end{aligned}
$$

[9, p. 131], where $F(x)$ and $F(x+i \pi)$ are in $\mathfrak{Q}^{2}$ by equation (2.15). To make an estimate of the integrals in this formula, it is convenient to split the interval $-\infty<x<\infty$ into three parts, $-\infty<x \leqq-X,-X<x<X$, and $X \leqq x<\infty$. Schwarz's inequality plus the fact that $F$ is in $\mathfrak{Q}^{2}$ implies that the contributions from $|x| \geqq X$ tend to 0 as $X$ tends to $\infty$. Given $X$, the contribution from $|x|<X$ tends to 0 as $u$ tends to $\pm \infty$. Thus, $F(u+i v)$ tends to 0 as $u$ tends to $\pm \infty$. It is easy to see that the limit is uniform in $v$ in any interval $0<v_{1} \leqq v \leqq v_{2}<\pi$. This proves the lemma.

LEMMA 2.5. If $f$ is in $\mathbb{S}$, and $f(r)$ and $f\left(r e^{i \pi}\right)$ are its boundary functions in the sense of Lemma 2.3, then

$$
\begin{gathered}
f(z)=(2 \pi i)^{-1} \int_{0}^{\infty}(r-z)^{-1} f(r) d r-(2 \pi i)^{-1} \int_{0}^{\infty}(r+z)^{-1} f\left(r e^{i \pi}\right) d r, \\
f(x+i y)=(y / \pi) \int_{0}^{\infty}\left[(r-x)^{2}+y^{2}\right]^{-1} f(r) d r \\
+(y / \pi) \int_{0}^{\infty}\left[(r+x)^{2}+y^{2}\right]^{-1} f\left(r e^{i \pi}\right) d r
\end{gathered}
$$

provided $\operatorname{Im} z>0$ and $y>0$.

Proof. Let $\Gamma$ be the boundary of the region bounded by the curves $\phi=\phi_{1}$, $\phi=\phi_{2}, r=r_{1}, r=r_{2}$, with $0<\phi_{1}<\phi_{2}<\pi$ and $0<r_{1}<r_{2}<\infty$. If $z$ is inside $\Gamma$,

$$
f(z)=(2 \pi i)^{-1} \int_{\Gamma}(w-z)^{-1} f(w) d w .
$$

Now let $r_{1}$ and $r_{2}$ tend to 0 and $\infty$, respectively. The integral (2.20) receives contributions from the circles $r=r_{1}$ and $r=r_{2}$. These tend to 0 by Lemma 2.4. The 
contributions from the rays $\phi=\phi_{1}$ and $\phi=\phi_{2}$ tend to limits owing to $f$ being in $\mathbb{S}$. Summarizing,

$$
\begin{aligned}
f(z)= & (2 \pi i)^{-1} \int_{0}^{\infty}\left(r \exp \left(i \phi_{1}\right)-z\right)^{-1} f\left(r \exp \left(i \phi_{1}\right)\right) \exp \left(i \phi_{1}\right) d r \\
& -(2 \pi i)^{-1} \int_{0}^{\infty}\left(r \exp \left(i \phi_{2}\right)-z\right)^{-1} f\left(r \exp \left(i \phi_{2}\right)\right) \exp \left(i \phi_{2}\right) d r .
\end{aligned}
$$

One can now let $\phi_{1}$ tend to 0 and $\phi_{2}$ tend to $\pi$. Schwarz's inequality and Lemma 2.3 then give equation (2.18).

The right-hand side of equation (2.20) vanishes if $z$ is replaced by $\bar{z}$. Hence, so do the right-hand sides of equations (2.21) and (2.18). Subtracting from equation (2.18) and writing $z=x+i y$ gives equation (2.19).

LEMMA 2.6. If $f$ is in $\mathfrak{S}$, it is in $\mathfrak{S}^{2}$.

Proof. It suffices to show that equation (2.1) is satisfied. Hence, owing to equation (2.19), it essentially suffices to show that the integral

$$
I=y^{2} \int_{-\infty}^{\infty} d x \int_{0}^{\infty} \int_{0}^{\infty}\left[(r-x)^{2}+y^{2}\right]^{-1}\left[(s-x)^{2}+y^{2}\right]^{-1}|f(r) f(s)| d r d s
$$

is bounded uniformly in $y$ for $y>0$. The integration over $x$ is straightforward, and gives

$$
I=2 \pi y \int_{0}^{\infty} \int_{0}^{\infty}\left[(r-s)^{2}+4 y^{2}\right]^{-1}|f(r) f(s)| d r d s .
$$

Writing $r-s=u$, we observe that $\int_{0}^{\infty}|f(r) f(r-u)| d r$ is bounded uniformly in $u$. Furthermore, the integral $\int_{-\infty}^{\infty} y\left(u^{2}+4 y^{2}\right)^{-1} d u$ is bounded uniformly in $y$, if $y>0$. Hence so is $I$. This takes care of the first term on the right in equation (2.19). The argument also applies to the second term. The triangle inequality now completes the proof.

THEOREM 2.7. The function classes $\mathfrak{S}^{2}$ and (S) are identical.

Proof. This follows from Lemmas 2.2 and 2.6.

COROLlaRY 2.8. If $f$ and $g$ are in $\mathfrak{S}^{2}$, then the integral

$$
\int_{0}^{\infty} f\left(r e^{i \phi}\right) g\left(r e^{i \phi}\right) e^{i \phi} d r
$$

regarded as a function of $\phi$, is constant in $0 \leqq \phi \leqq \pi$.

Proof. Since $\mathfrak{S}^{2}$ is the same class as $\mathscr{S}$, the integral converges. Now let $\Gamma$ be the contour introduced in the proof of Lemma 2.5. Then

$$
\int_{\Gamma} f(z) g(z) d z=0 .
$$

Lemma 2.4 makes it possible to let $r_{1}$ and $r_{2}$ tend to 0 and $\infty$, respectively. This gives the required result for $0<\phi<\pi$. Using Lemma 2.5, one can let $\phi_{1}$ and $\phi_{2}$ 
tend to 0 and $\pi$, respectively, and so one can also cover the limiting cases $\phi=0, \pi$. It was already indicated in $\S 1.2$ that this result is useful in applications.

2.3. Mellin transforms. According to the Paley-Wiener theorem, $f$ belongs to $\mathfrak{S}^{2}$ if and only if the Fourier transform of its boundary function vanishes for negative arguments $[2$, p. 131], $[5$, p. 8$],[9$, p. 128]. There is a similar result on Fourier transforms of functions analytic in a strip. Now remember that Lemma 2.3 is based on the transformation $e^{w}=r e^{i \phi}$, which maps a strip in the $w$-plane onto the upper half $r e^{i \phi}$-plane. The transformation takes the Fourier transform into the Mellin transform. As a result, there is a Mellin-analogue of the Paley-Wiener theorem. We now turn to the proof of this.

THEOREM 2.9. A complex-valued function $f$ defined on the upper half-plane belongs to the class $\mathfrak{S}^{2}$ if and only if it is an inverse Mellin transform according to

$$
f\left(r e^{i \phi}\right)=(2 \pi)^{-1 / 2} \int_{-\infty}^{\infty} f(t)\left(r e^{i \phi}\right)^{-i t-1 / 2} d t,
$$

with some function $f(t)$ satisfying

$$
\int_{-\infty}^{\infty}\left(1+e^{2 \pi t}\right)|\boldsymbol{f}(t)|^{2} d t<\infty
$$

Proof. The function $F(w)$ which corresponds to $f\left(r e^{i \phi}\right)$ according to the proof of Lemma 2.3 is of the form

$$
F(w)=(2 \pi)^{-1 / 2} \int_{-\infty}^{\infty} f(t) e^{-i w t} d t,
$$

where $f(t)$ must satisfy equation (2.27). This follows from the theory of functions that are analytic in a strip [9, p. 131]. The relation (2.13) between $F$ and $f$ gives equation (2.26), showing that the condition of the theorem is necessary.

Now suppose that $f(t)$ is given and satisfies equation (2.27). Consider the righthand side of equation (2.26). If $0<\phi<\pi$, Schwarz's inequality and equation (2.27) readily show that the integral in equation (2.26) converges absolutely. Since the integrand is analytic, so is the integral [1, p. 257]. Thus, $f$ as defined by equation (2.26) is analytic in the upper half-plane. Now observe that $f\left(r e^{i \phi}\right)$, regarded as a function of $r$, is the inverse Mellin transform of $f(t) \exp \left(\phi t-\frac{1}{2} i \phi\right)$. Since the latter function is square-integrable, again by equation (2.27), so is $f\left(r e^{i \phi}\right)$. It is easy to see that the integrated square is bounded uniformly in $\phi$ for $0<\phi<\pi$. Hence, $f$ is in (s). But $\mathfrak{B S}$ is nothing but $\mathfrak{S}^{2}$, and so the condition of the theorem is sufficient. This completes the proof.

REMARK 2.10. Taking Mellin transforms in Theorem 2.9 gives the result that $f$ is in $\mathfrak{S}^{2}$ if and only if its Mellin transform is of the form

$$
f(t, \phi)=(2 \pi)^{-1 / 2} \int_{0}^{\infty} f\left(r e^{i \phi}\right) r^{i t-1 / 2} d r=e^{\phi t-i \phi / 2} f(t),
$$

$f(t)$ satisfying equation (2.27). 
REMARK 2.11. It only requires a minor extension of Theorem 2.9 to prove that a function $f(r)$ which is defined on $r \geqq 0$ and is square-integrable is the boundary value, as $\phi$ tends to 0 , of a function $f$ in $\mathfrak{S}^{2}$ if and only if its Mellin transform $f(t)$ satisfies equation (2.27). Given $f(r)$, one can find $f(t)$. Next, equation (2.26) yields $f\left(r e^{i \phi}\right)$, and so one can actually construct the function in the upper half-plane from its values on a mere part of the boundary.

2.4. The class $\mathfrak{S}^{2}$ as a Hilbert space. If an inner product is defined according to equation (2.4), the class $\mathfrak{S}^{2}$ becomes a Hilbert space. For the applications which we have in mind, it is useful to compare the Hilbert space $\mathfrak{S g}^{2}$ with a space of Mellin transforms of functions in $\mathfrak{S}^{2}$. This is facilitated by the following definitions.

Definition 2.12. For any $f$ in $\mathfrak{S}^{2}$, the corresponding boldface letter $\boldsymbol{f}$ denotes the Mellin transform $f(t, \phi)$ of the function $f\left(r e^{i \phi}\right)$, in the sense of equation (2.29).

Definition 2.13. The class of functions

$$
f(t, \phi)=e^{\phi t-i \phi / 2} f(t)
$$

defined on $-\infty<t<\infty, 0 \leqq \phi \leqq \pi$ which have the property that

$$
\int_{-\infty}^{\infty} e^{2 \phi t}|\boldsymbol{f}(t)|^{2} d t
$$

exists and is bounded, uniformly in $\phi$ for $0 \leqq \phi \leqq \pi$, is denoted by $\mathfrak{Q}^{2}$. With the inner product

$$
(\boldsymbol{f}, \boldsymbol{g})=\int_{-\infty}^{\infty}\left(1+e^{2 \pi t}\right) \boldsymbol{f}(t) \bar{g}(t) d t
$$

this class is a Hilbert space. This is also denoted by $\mathfrak{\mathfrak { G }}^{2}$.

There is an intimate relation between the spaces $\mathfrak{S}^{2}$ and $\mathfrak{G}^{2}$, which is used throughout the rest of this paper. It is expressed by the following theorem.

THEOREM 2.14. The mapping $f \rightarrow f$ generated by the Mellin transform according to equation (2.29) is a unitary mapping of $\mathfrak{S}^{2}$ onto $\mathfrak{\mathfrak { Q }}^{2}$.

Proof. Since Theorem 2.9 and Remark 2.10 already state that the mapping is onto, it suffices to show that it preserves inner products. If $g$ is in $\mathfrak{S}^{2}$, then the Mellin transform of $\bar{g}\left(r e^{i \phi}\right)$ is $\bar{g}(-t) \exp \left(-\phi t+\frac{1}{2} i \phi\right)$, by equation (2.29). Thus, by general formulas for Mellin transforms,

$$
\begin{aligned}
& \int_{0}^{\infty} f(r) \bar{g}(r) d r+\int_{0}^{\infty} f\left(r e^{i \pi}\right) \bar{g}\left(r e^{i \pi}\right) d r \\
&=\int_{-\infty}^{\infty} \boldsymbol{f}(t) \overline{\boldsymbol{g}}(t) d t+\int_{-\infty}^{\infty} e^{\pi t-i \pi / 2} f(t) e^{\pi t+i \pi / 2} \overline{\boldsymbol{g}}(t) d t
\end{aligned}
$$

The left-hand side is $(f, g)$ and the right-hand side is $(f, g)$. This proves the theorem. 
REMARK 2.15. In addition to equation (2.33), the general theory of Mellin transforms also implies that, if $0 \leqq \phi \leqq \pi$,

$$
\int_{0}^{\infty}\left|f\left(r e^{i \phi}\right)\right|^{2} d r=\int_{-\infty}^{\infty} e^{2 \phi t}|f(t)|^{2} d t
$$

Since the right-hand side of this expression is a convex function of $\phi$, so is the left side. In other words, if $0 \leqq \phi \leqq \pi$,

$$
\int_{0}^{\infty}\left|f\left(r e^{i \phi}\right)\right|^{2} d r \leqq(1-\phi / \pi) \int_{0}^{\infty}|f(r)|^{2} d r \dot{+}(\phi / \pi) \int_{0}^{\infty}\left|f\left(r e^{i \pi}\right)\right|^{2} d r .
$$

The equality sign occurs only if $f \equiv 0$. Denoting the norm of $f$ by $\|f\|$ yields

$$
\int_{0}^{\infty}\left|f\left(r e^{i \phi}\right)\right|^{2} d r \leqq\|f\|^{2}
$$

Applying Hölder's inequality to the right-hand side of equation (2.34) shows that $\log \int_{0}^{\infty}\left|f\left(r e^{i \phi}\right)\right|^{2} d r$ is also a convex function of $\phi$.

We conclude this section with a definition, followed by a theorem which says that the class $\mathfrak{S}^{2}$ is actually very large.

Definition 2.16. The set of all complex-valued functions $g(r)$ which are defined and square-integrable on the half-line $r \geqq 0$ is denoted by $\mathfrak{Q}^{2}$. The set of all complexvalued functions $\boldsymbol{g}(t)$ which are defined and square-integrable on $-\infty<t<\infty$ is denoted by $\mathbf{2}^{2}$.

THEOREM 2.17. Given a function $g(r)$ in $\mathfrak{L}^{2}$, a fixed angle $\psi$ in $0 \leqq \psi \leqq \pi$, and a positive $\varepsilon$, there is a function $f\left(r e^{i \phi}\right)$ in $\mathfrak{S}^{2}$ such that

$$
\int_{0}^{\infty}\left|f\left(r e^{i \psi}\right)-g(r)\right|^{2} d r<\varepsilon
$$

Proof. Let $g(t)$ be the Mellin transform of $g(r)$. Given $\varepsilon$, there is a positive $T$ such that

$$
\int_{-\infty}^{-T}|g(t)|^{2} d t+\int_{T}^{\infty}|g(t)|^{2} d t<\varepsilon
$$

Now define $f(t)$ by

$$
\begin{aligned}
f(t) & =e^{-\psi t+i \psi / 2} g(t) & & (-T<t<T), \\
& =0 & & (|t|>T) .
\end{aligned}
$$

Next, consider the function $f(t) \exp \left(\phi t-\frac{1}{2} i \phi\right)$. This is a function $f(t, \phi)$ in $\mathfrak{q}^{2}$. Also, $f(t, \psi)$ vanishes for large $|t|$ but is equal to $g(t)$ otherwise. More precisely,

$$
\int_{-\infty}^{\infty}|\boldsymbol{f}(t, \psi)-\boldsymbol{g}(t)|^{2} d t<\varepsilon
$$

Taking inverse Mellin transforms gives a function $f\left(r e^{i \phi}\right)$ in $\mathfrak{S}^{2}$. It also takes equation (2.40) into equation (2.37), showing that $f$ satisfies the conditions of the theorem. 
COROLlARY 2.18. The boundary values $f(r)$ of functions $f\left(r e^{i \phi}\right)$ in $\mathfrak{S}^{2}$ are dense in $\mathfrak{R}^{2}$.

\section{A Hilbert space of integral operators.}

3.1. The function class $\mathfrak{R}$. If the space $\mathfrak{H}^{2}$ is discussed in the language developed above, it becomes natural to consider a certain class of integral operators on $\mathfrak{S}^{2}$ which is studied in this and the next few sections. We start by formally defining a class $\mathfrak{R}$ of functions $K\left(r, r^{\prime}, \phi\right)$. Next, we show that the functions in $\Re$ can conveniently be characterized in terms of their Mellin transforms. From this it then follows that one can define an inner product which makes $\mathfrak{R}$ a Hilbert space. This function space provides the starting point for a corresponding space of operators, which is studied in the next section.

Definition 3.1. Let $K\left(r, r^{\prime}, \phi\right)$ be a function defined on $0<r<\infty, 0<r^{\prime}<\infty$, $0<\phi<\pi$, and suppose that

(1) The integral

$$
\int_{0}^{\infty} \int_{0}^{\infty}\left|K\left(r, r^{\prime}, \phi\right)\right|^{2} d r d r^{\prime}
$$

exists and is bounded, uniformly in $\phi$ for $0<\phi<\pi$.

(2) The function

$$
(K f)(r, \phi)=\int_{0}^{\infty} K\left(r, r^{\prime}, \phi\right) f\left(r^{\prime} e^{i \phi}\right) e^{i \phi} d r^{\prime}
$$

depends only on $r e^{i \phi}$ and is in $\mathfrak{S}^{2}$ whenever $f$ is in $\mathfrak{S}^{2}$. The class of all functions $K$ with these properties is denoted by $\Omega$.

LEMMA 3.2. If $K$ is in $\mathfrak{R}$, then its Mellin transform is of the form

$$
\begin{aligned}
K\left(t, t^{\prime}, \phi\right) & =(2 \pi)^{-1} \int_{0}^{\infty} \int_{0}^{\infty} K\left(r, r^{\prime}, \phi\right) r^{i t-1 / 2}\left(r^{\prime}\right)^{-i t^{\prime}-1 / 2} d r d r^{\prime} \\
& =e^{\phi t-\phi t^{\prime}-i \phi} K\left(t, t^{\prime}\right),
\end{aligned}
$$

with some function $K\left(t, t^{\prime}\right)$ satisfying

$$
\int_{-\infty}^{\infty} \int_{-\infty}^{\infty}\left(1+e^{2 \pi t-2 \pi t^{\prime}}\right)\left|K\left(t, t^{\prime}\right)\right|^{2} d t d t^{\prime}<\infty
$$

Proof. By property (1) of Definition 3.1, the integral

$$
I=\int_{0}^{\infty} \int_{0}^{\infty} g\left(r e^{i \phi}\right) K\left(r, r^{\prime}, \phi\right) f\left(r^{\prime} e^{i \phi}\right) e^{2 i \phi} d r d r^{\prime}
$$

converges whenever $f$ and $g$ are in $\mathfrak{S}^{2}$. By property (2) and Corollary 2.8, it does not depend on $\phi$. The function $K$ has a double Mellin transform by property (1). Let this be the function $K\left(t, t^{\prime}, \phi\right)$ as defined by the second member of equation (3.3). Then

$$
I=\int_{-\infty}^{\infty} \int_{-\infty}^{\infty} g(-t) K\left(t, t^{\prime}, \phi\right) f\left(t^{\prime}\right) e^{-\phi t+\phi t^{\prime}+i \phi} d t d t^{\prime}
$$


Now, $\boldsymbol{f}$ and $\boldsymbol{g}$ may be any two functions which are square-integrable and vanish outside bounded intervals. Also, $\boldsymbol{K}$ is square-integrable. Since $I$ does not depend on $\phi$, it follows that the integrand does not depend on $\phi$. This gives equation (3.3).

To get equation (3.4), observe that the integral of $\left|K\left(t, t^{\prime}, \phi\right)\right|^{2}$ with respect to $t$ and $t^{\prime}$ is equal to the expression (3:1). Since this is bounded on $0<\phi<\pi$ by assumption, so is the integral of $|\boldsymbol{K}|^{2}$. The relation (3.4) follows from this by an obvious limiting process.

REMARK 3.3. Our notation is chosen such that

$$
\begin{aligned}
(\boldsymbol{K} f)(t, \phi) & =(2 \pi)^{-1 / 2} \int_{0}^{\infty}(K f)(r, \phi) r^{i t-1 / 2} d r \\
& =e^{\phi t-i \phi / 2} \int_{-\infty}^{\infty} K\left(t, t^{\prime}\right) f\left(t^{\prime}\right) d t^{\prime}
\end{aligned}
$$

Corollary 3.4. If $K$ is in $\mathfrak{\Omega}$ then the function

$$
(g K)\left(r^{\prime}, \phi\right)=\int_{0}^{\infty} g\left(r e^{i \phi}\right) K\left(r, r^{\prime}, \phi\right) e^{i \phi} d r
$$

depends only on $r^{\prime} e^{i \phi}$ and is in $\mathfrak{S}^{2}$ whenever $g$ is in $\mathfrak{S}^{2}$.

Proof. Owing to Lemma 3.2, the Mellin transform of $(g K)$ is equal to

$$
e^{\phi t^{\prime}-i \phi / 2} \int_{-\infty}^{\infty} g(-t) K\left(t,-t^{\prime}\right) d t
$$

and is thus of the form required by Remark 2.10. Since $\boldsymbol{g}$ is in $\mathfrak{\mathscr { Q }}^{2}$ and $\boldsymbol{K}$ satisfies equation (3.4), it is easy to check that the integral in equation (3.9) has the integrability property (2.27). Hence, $(g K)$ is in $\mathfrak{S}^{2}$ by Remark 2.10 .

By using Mellin transforms, one can easily prove a number of statements on functions in $\mathfrak{A}$ that are analogous to properties of functions in $\mathfrak{S}^{2}$. We proceed to give a summary of these.

TheOREM 3.5. A function $K\left(r, r^{\prime}, \phi\right)$ defined on $0<r<\infty, 0<r^{\prime}<\infty, 0<\phi<\pi$ is in $\Re$ if and only if it is of the form

$$
K\left(r, r^{\prime}, \phi\right)=(2 \pi)^{-1} \int_{-\infty}^{\infty} \int_{-\infty}^{\infty} K\left(t, t^{\prime}\right)\left(r e^{i \phi}\right)^{-i t-1 / 2}\left(r^{\prime} e^{i \phi}\right)^{i t^{\prime}-1 / 2} d t d t^{\prime}
$$

with some function $\mathrm{K}$ satisfying equation (3.4).

Proof. It follows from Lemma 3.2 that the condition of the theorem is necessary. Now suppose that we are given a function $K\left(t, t^{\prime}\right)$ satisfying equation (3.4). For $0<\phi<\pi$, the right-hand side of equation (3.10) converges absolutely and thus defines a function $K\left(r, r^{\prime}, \phi\right)$. Owing to equation (3.4), the function $|K|^{2}$ is integrable, hence $K$ has property (1) of Definition 3.1.

For any $f$ in $\mathfrak{S}^{2}$, the integral (3.2) for $(K f)$ converges absolutely for almost every $r$ and is a square-integrable function of $r$. By studying its Mellin transform as in 
the proof of Corollary 3.4, it readily follows that $(K f)$ depends only on $r e^{i \phi}$ and is in $\mathfrak{S}^{2}$. Hence, $K$ is in $\Re$, as we wished to show.

LEMMA 3.6. If $K$ is in $\AA$, then there are boundary functions $K\left(r, r^{\prime}, 0\right)$ and $K\left(r, r^{\prime}, \pi\right)$ such that

$$
\lim _{\phi \rightarrow \psi} \int_{0}^{\infty} \int_{0}^{\infty}\left|K\left(r, r^{\prime}, \phi\right)-K\left(r, r^{\prime}, \psi\right)\right|^{2} d r d r^{\prime}=0 \quad(\psi=0, \pi) .
$$

If $f$ is in $\mathfrak{S}^{2}$ and $(K f)$ is defined by equation (3.2), then the boundary values of $(K f)$ satisfy

$$
\lim _{\phi \rightarrow \psi} \int_{0}^{\infty}\left|(K f)\left(r e^{i \phi}\right)-\int_{0}^{\infty} K\left(r, r^{\prime}, \psi\right) f\left(r^{\prime} e^{i \psi}\right) e^{i \psi} d r^{\prime}\right|^{2} d r=0 \quad(\psi=0, \pi) .
$$

Proof. Consider the Mellin transform $K$ of $K$. With the help of Lemma 3.2, it is easy to see that this has mean-square limits as $\phi$ tends to 0 or to $\pi$. In particular, it tends to $K\left(t, t^{\prime}\right)$ as $\phi$ tends to 0 . Now let $K\left(r, r^{\prime}, 0\right)$ be the inverse Mellin transform of $K\left(t, t^{\prime}\right)$. Then $K\left(r, r^{\prime}, 0\right)$ satisfies equation (3.11).

Since $(K f)$ belongs to $\mathfrak{S}^{2}$, it must tend to boundary values as $\phi$ tends to 0 or to $\pi$. Given $K\left(r, r^{\prime}, 0\right)$ as defined above, it is not difficult to check that the Mellin transform of $(K f)$ tends to the Mellin transform of the second term on the left in equation (3.12). This suffices to prove equation (3.12) for $\phi$ tending to 0 .

The case of $\phi$ tending to $\pi$ can be discussed in the same way. This completes the proof of the lemma.

Definition 3.7. The class of functions

$$
K\left(t, t^{\prime}, \phi\right)=e^{\phi t-\phi t^{\prime}-i \phi} K\left(t, t^{\prime}\right),
$$

defined on $-\infty<t<\infty,-\infty<t^{\prime}<\infty, 0 \leqq \phi \leqq \pi$ and such that $K\left(t, t^{\prime}\right)$ satisfies equation (3.4), is denoted by $\AA$. With the inner product

$$
(K, L)=\int_{-\infty}^{\infty} \int_{-\infty}^{\infty}\left(1+e^{2 \pi t-2 \pi t^{\prime}}\right) K\left(t, t^{\prime}\right) \bar{L}\left(t, t^{\prime}\right) d t d t^{\prime}
$$

this class is a Hilbert space. This is also denoted by $\$$.

THEOREM 3.8. With the inner product

$$
(K, L)=\int_{0}^{\infty} \int_{0}^{\infty}\left[K\left(r, r^{\prime}, 0\right) \bar{L}\left(r, r^{\prime}, 0\right)+K\left(r, r^{\prime}, \pi\right) \bar{L}\left(r, r^{\prime}, \pi\right)\right] d r d r^{\prime}
$$

the class $\mathfrak{\Omega}$ becomes a Hilbert space. The mapping $K \rightarrow K$ generated by the Mellin transform according to equation (3.3) is a unitary mapping of $\Re$ onto $\$$.

Proof. Theorem 3.5 states that the mapping is onto. If $(K, L)$ and $(K, L)$ are defined by equations (3.15) and (3.14), then

$$
(K, L)=(K, L)
$$

This can be shown as in the proof of Theorem 2.14. Since it is obvious that is a Hilbert space, equation (3.16) implies that $(K, L)$ has all the properties of an inner 
product. It also follows that $\AA$ becomes complete under the norm which derives from the inner product (3.15). Hence, $\mathfrak{R}$ becomes a Hilbert space. The Mellin transform maps this onto and preserves inner products. Hence, the Mellin transform is unitary.

3.2. Operators on $\mathfrak{S}^{2}$. From now on, the Mellin transform is denoted by $\Gamma$, and is regarded as a unitary operator on $\mathfrak{S}^{2}$ onto $\mathfrak{\mathbb { E }}^{2}$. Thus,

$$
\Gamma f\left(r e^{i \phi}\right)=f(t, \phi) .
$$

If $B$ is any bounded linear operator on $\mathfrak{S}^{2}$, then $(B f, g)$ is a bilinear functional on $\mathfrak{S}^{2}$. Now,

$$
(B f, g)=\left(B \Gamma^{-1} f, \Gamma^{-1} g\right)
$$

hence $(B f, g)$ can also be regarded as a bilinear functional on $\mathfrak{\mathbb { E }}^{2}$. This defines an operator on $\mathfrak{\mathscr { Q }}^{2}$ which we denote by $\boldsymbol{B}$. In other words,

$$
\Gamma B \Gamma^{-1}=\boldsymbol{B} \text {. }
$$

The norm of $B$ is denoted by $\|B\|$.

Now let $K$ be any function in $\Re$. The relation

$$
K f\left(r e^{i \phi}\right)=\int_{0}^{\infty} K\left(r, r^{\prime}, \phi\right) f\left(r^{\prime} e^{i \phi}\right) e^{i \phi} d r^{\prime}
$$

then defines a linear operator with domain $\mathfrak{S}^{2}$. This is denoted by $K$, the set of all operators $K$ obtained in this way being denoted by $\Re$. Thus, $\Re$ is now both a function class and a class of operators. Given any $K$ in $\Re$, it follows from Remark 3.3 that the corresponding operator $K$ is an integral operator on $\mathfrak{\mathcal { Q }}^{2}$ acting according to

$$
K \boldsymbol{f}(t, \phi)=e^{\phi t-i \phi / 2} \int_{-\infty}^{\infty} K\left(t, t^{\prime}\right) f\left(t^{\prime}\right) d t^{\prime}=\int_{-\infty}^{\infty} e^{\phi t} K\left(t, t^{\prime}\right) e^{-\phi t^{\prime}} f\left(t^{\prime}, \phi\right) d t^{\prime}
$$

By Theorem 3.8, $\mathfrak{A}$ and $\AA$ can be regarded as Hilbert spaces of operators. Referring to equations (3.14) and (3.15), we define the $\kappa$-norm by

$$
\begin{aligned}
\kappa(K) & =\left[\int_{0}^{\infty} \int_{0}^{\infty}\left(\left|K\left(r, r^{\prime}, 0\right)\right|^{2}+\left|K\left(r, r^{\prime}, \pi\right)\right|^{2}\right) d r d r^{\prime}\right]^{1 / 2} \\
& =\kappa(K)=\left[\int_{-\infty}^{\infty} \int_{-\infty}^{\infty}\left(1+e^{2 \pi t-2 \pi t^{\prime}}\right)\left|K\left(t, t^{\prime}\right)\right|^{2} d t d t^{\prime}\right]^{1 / 2}
\end{aligned}
$$

The relation

$$
\int_{0}^{\infty} \int_{0}^{\infty}\left|K\left(r, r^{\prime}, \phi\right)\right|^{2} d r d r^{\prime}=\int_{-\infty}^{\infty} \int_{-\infty}^{\infty} e^{2 \phi t-2 \phi t^{\prime}}\left|K\left(t, t^{\prime}\right)\right|^{2} d t d t^{\prime} \leqq[\kappa(K)]^{2}
$$

is analogous to equations (2.34) and (2.36).

LEMMA 3.9. If $K$ is any operator in $\AA$, then

$$
\|K\| \leqq \kappa(K) \text {. }
$$


Proof. This follows from equation (3.20) with Schwarz's inequality and equation (3.23).

3.3. The Schmidt class on $\mathfrak{S}^{2}$. Our emphasis on functions being square-integrable suggests that we compare the class $\mathfrak{A}$ with the Schmidt class on $\mathfrak{S}^{2}$. The Schmidt class is denoted by $\mathfrak{S}$. It is the class of all operators $S$ on $\mathfrak{S}^{2}$ which admit polar decompositions of the form

$$
S f=\sum_{n} \sigma_{n} a_{n}\left(f, b_{n}\right),
$$

where $\left\{a_{n}\right\}$ and $\left\{b_{n}\right\}$ are orthonormal sets in $\mathscr{S}^{2}$, the quantities $\sigma_{n}$ are positive numbers, and $\sum_{n} \sigma_{n}^{2}$ converges [6, p. 33]. The Schmidt norm is defined by

$$
\sigma(S)=\left[\sum_{n} \sigma_{n}^{2}\right]^{1 / 2}
$$

In an obvious notation, the corresponding operator $S$ acts according to

$$
S f(t, \phi)=e^{\phi t-i \phi / 2} \sum_{n} \sigma_{n} a_{n}(t) \int_{-\infty}^{\infty}\left(1+e^{2 \pi t^{\prime}}\right) f\left(t^{\prime}\right) \bar{b}\left(t^{\prime}\right) d t^{\prime}
$$

and is thus of the form

$$
S f(t, \phi)=e^{\phi t-\imath \phi / 2} \int_{-\infty}^{\infty} S\left(t, t^{\prime}\right) f\left(t^{\prime}\right) d t^{\prime}=\int_{-\infty}^{\infty} e^{\phi t} S\left(t, t^{\prime}\right) e^{-\phi t^{\prime}} f\left(t^{\prime}, \phi\right) d t^{\prime},
$$

with

$$
S\left(t, t^{\prime}\right)=\sum_{n} \sigma_{n} a_{n}(t) \bar{b}_{n}\left(t^{\prime}\right)\left(1+e^{2 \pi t^{\prime}}\right) .
$$

Since $\left\{\boldsymbol{a}_{n}\right\}$ and $\left\{\boldsymbol{b}_{n}\right\}$ are orthonormal sets in $\mathfrak{\mathscr { \Psi }}^{2}$, it readily follows that

$$
\sigma(S)=\left[\int_{-\infty}^{\infty} \int_{-\infty}^{\infty}\left(1+e^{2 \pi t}\right)\left|S\left(t, t^{\prime}\right)\right|^{2}\left(1+e^{2 \pi t^{\prime}}\right)^{-1} d t d t^{\prime}\right]^{1 / 2}
$$

This leads to the following theorem.

THEOREM 3.10. An operator $S$ on $\mathfrak{S}^{2}$ belongs to $S$ if and only if its Mellin transform $S$ acts according to equation (3.28), with some function $S\left(t, t^{\prime}\right)$ satisfying equation (3.30).

Proof. It was shown above that the condition is necessary. Now suppose that we are given an operator $S$ such that its Mellin transform $S$ is of the form (3.28) and satisfies equation (3.30). From equation (3.30), it follows that the function

$$
R\left(t, t^{\prime}\right)=\left(1+e^{2 \pi t}\right)^{1 / 2} S\left(t, t^{\prime}\right)\left(1+e^{2 \pi t^{\prime}}\right)^{-1 / 2}
$$

is a square-integrable function of $t$ and $t^{\prime}$, and is therefore the kernel of an operator in the Schmidt class on $\mathbf{2}^{2}$. Hence, $\boldsymbol{R}$ has a decomposition

$$
R\left(t, t^{\prime}\right)=\sum_{n} \rho_{n} c_{n}(t) \bar{d}_{n}\left(t^{\prime}\right)
$$


in terms of orthonormal sets $\left\{\boldsymbol{c}_{n}\right\}$ and $\left\{\boldsymbol{d}_{n}\right\}$ in $\mathbf{2}^{2}$, the sum $\sum_{n} \rho_{n}^{2}$ converging. Now, if $\left\{\boldsymbol{c}_{n}\right\}$ is orthonormal in $\mathbf{2}^{2}$, then $\left\{\left(1+e^{2 \pi t}\right)^{-1 / 2} \boldsymbol{c}_{n}\right\}$ is an orthonormal set in $\mathfrak{\mathfrak { y }}^{2}$. Let this be denoted by $\left\{\boldsymbol{a}_{n}\right\}$, and denote the set corresponding to $\left\{\boldsymbol{d}_{n}\right\}$ by $\left\{\boldsymbol{b}_{n}\right\}$. Solving equation (3.31) for $S\left(t, t^{\prime}\right)$ then yields a function $S\left(t, t^{\prime}\right)$ which is exactly of the form (3.29), with $\sigma_{n}=\rho_{n}$. The corresponding operator $S$ must act according to equation (3.25), the sum $\sum_{n} \sigma_{n}^{2}$ converging. Hence, $S$ is in $\subseteq$. This completes the proof of the theorem.

The next theorem gives the desired comparison between the classes $\Re$ and $\subseteq$.

THEOREM 3.11. The class $\mathfrak{\Re}$ is a proper subclass of the Schmidt class $\mathfrak{S}$. If $K$ is an operator in $\AA$, then

$$
\sigma(K) \leqq \kappa(K) .
$$

Proof. If $K$ is any operator in $\Re$, then $K$ acts according to equation (3.21), the $\kappa$-norm being given by equation (3.22). Now notice the similarity between equations (3.21) and (3.28). Since

$$
\left(1+e^{2 \pi t}\right)\left(1+e^{2 \pi t^{\prime}}\right)^{-1} \leqq 1+e^{2 \pi t-2 \pi t^{\prime}},
$$

the integral (3.30) for $\sigma(K)$ converges and does not exceed $\kappa(K)$. Hence, equation (3.33) is satisfied. By Theorem $3.10, K$ is in $\subseteq$, and the quantity $\sigma(K)$ is the Schmidt norm of $K$. This shows that the class $\Omega$ is contained in $\mathfrak{S}$.

It remains to prove that $\AA$ is a proper subclass of $\mathfrak{S}$. To see this, consider a kernel $S\left(t, t^{\prime}\right)$ of the form

$$
S\left(t, t^{\prime}\right)=a(t) \bar{b}\left(t^{\prime}\right)\left(1+e^{2 \pi t^{\prime}}\right),
$$

where $\boldsymbol{a}$ and $\boldsymbol{b}$ are in $\mathfrak{\mathfrak { g }}^{2}$. This corresponds to an operator $S$ of rank 1 . Whether this is in $\Re$ is determined by the integral

$$
\int_{-\infty}^{\infty} \int_{-\infty}^{\infty}\left(1+e^{2 \pi t-2 \pi t^{\prime}}\right)\left|\boldsymbol{a}(t) \boldsymbol{b}\left(t^{\prime}\right)\right|^{2}\left(1+e^{2 \pi t^{\prime}}\right)^{2} d t d t^{\prime} .
$$

Given the fact that $\boldsymbol{a}$ and $\boldsymbol{b}$ are in $\mathfrak{\mathscr { \mathfrak { S } }}^{2}$, the integral converges if and only if $\boldsymbol{b}(t) e^{-\pi t}$ and $\boldsymbol{b}(t) e^{2 \pi t}$ are in $\mathbf{Q}^{2}$. Thus, there are many examples of operators of rank 1 which do not belong to $\Re$. This makes it obvious that $\mathbb{\Omega}$ is a proper subclass of $\mathbb{S}$ and thereby completes the proof of the theorem.

We conclude this section with a result analogous to Theorem 2.17. It says that $\mathfrak{A}$ is dense in $\mathfrak{S}$ in the Schmidt norm.

THEOREM 3.12. Given an operator $S$ in $\mathfrak{S}$, there is a sequence of operators $K_{T}$ in 爪 such that

$$
\lim _{T \rightarrow \infty} \sigma\left(S-K_{T}\right)=0 .
$$

Proof. If $-T<t^{\prime}<T$, it is easy to see that

$$
1+e^{2 \pi t-2 \pi t^{\prime}} \leqq\left(1+e^{2 \pi T}\right)\left(1+e^{2 \pi t}\right)\left(1+e^{2 \pi t^{\prime}}\right)^{-1} .
$$


Now let $S$ be an operator in $\subseteq$ and consider the function $S\left(t, t^{\prime}\right)$ defined by equation (3.28). For any $T>0$, define

$$
\begin{aligned}
K_{T}\left(t, t^{\prime}\right) & =S\left(t, t^{\prime}\right) & & \left(-T<t^{\prime}<T\right), \\
& =0 & & \left(\left|t^{\prime}\right|>T\right) .
\end{aligned}
$$

It is obvious that the operator $K_{T}$ associated with $K_{T}\left(t, t^{\prime}\right)$ is in $\mathfrak{S}$. By equation (3.38) and Theorem 3.5, it is also in $\Re$. Furthermore, by equation (3.30)

$$
\sigma\left(S-K_{T}\right)=\left[\int_{\left|t^{\prime}\right|>T} d t^{\prime} \int_{-\infty}^{\infty}\left(1+e^{2 \pi t}\right)\left|S\left(t, t^{\prime}\right)\right|^{2}\left(1+e^{2 \pi t^{\prime}}\right)^{-1} d t d t^{\prime}\right]^{1 / 2}
$$

which tends to 0 as $T$ tends to $\infty$, owing to $S$ being in $\subseteq$. Hence, $K_{T}$ tends to $S$ in the Schmidt norm. This proves the theorem.

\section{A Banach algebra of linear operators.}

4.1. Norm ideals. If $S$ is in the Schmidt class $\subseteq$ and $B$ is any bounded linear operator with norm $\|B\|$, it is well known $[6$, p. 30] that both $B S$ and $S B$ are in $\subseteq$, the Schmidt norms satisfying

$$
\sigma(B S) \leqq\|B\| \sigma(S), \quad \sigma(S B) \leqq\|B\| \sigma(S) .
$$

In the particular case of a unitary operator $U$, one has

$$
\sigma(U S)=\sigma(S U)=\sigma(S) .
$$

In the following, the algebra of all bounded linear operators on $\mathfrak{S}^{2}$ is denoted by $\mathfrak{B}$. In Schatten's terminology [6, p. 54], $\mathfrak{S}$ is a norm ideal in $\mathfrak{B}$.

The class $K$ is not an ideal in $\mathfrak{B}$. For example, consider the operator $U$ defined by

$$
U f\left(r e^{i \phi}\right)=i\left(r e^{i \phi}\right)^{-1} f\left(1 / r e^{i \phi-i \pi}\right) .
$$

It is easily seen that $U^{2}=1$. In fact, $U$ is unitary and selfadjoint. It corresponds to the operator $\boldsymbol{U}$ on $\mathfrak{\mathbb { Q }}^{2}$ given by

$$
\boldsymbol{U} \boldsymbol{f}(t, \phi)=e^{\phi t-i \phi / 2-\pi t} f(-t) .
$$

Now let $K$ be an operator in $\Re$ and consider the function $K\left(t, t^{\prime}\right)$ which satisfies

$$
K f(t)=\int_{-\infty}^{\infty} K\left(t, t^{\prime}\right) f\left(t^{\prime}\right) d t^{\prime} .
$$

Remember that $f(t)$ is the same as $f(t, 0)$. With equation (4.4) it follows that

$$
\begin{aligned}
& \boldsymbol{U K f}(t)=\int_{-\infty}^{\infty} e^{-\pi t} K\left(-t, t^{\prime}\right) f\left(t^{\prime}\right) d t^{\prime}, \\
& \boldsymbol{K U} \boldsymbol{f}(t)=\int_{-\infty}^{\infty} K\left(t,-t^{\prime}\right) e^{\pi t^{\prime}} f\left(t^{\prime}\right) d t^{\prime}
\end{aligned}
$$

In order that $U K$ and $K U$ be in $\Re$, it is necessary and sufficient that

$$
\int_{-\infty}^{\infty} \int_{-\infty}^{\infty}\left(e^{2 \pi t}+e^{-2 \pi t^{\prime}}\right)\left|K\left(t, t^{\prime}\right)\right|^{2} d t d t^{\prime}<\infty,
$$


by Theorem 3.5. But $K$ being in $\Omega$ merely requires that the integral (3.4) converges, and so there are numerous examples of operators in $\AA$ such that $U K$ and $K U$ are not in $\Re$.

It is crucial in the foregoing example that $U f\left(r e^{i \phi}\right)$ depends on values taken by $f\left(r e^{i \downarrow}\right)$ for arguments $\psi$ other than $\phi$. Indeed, $U f\left(r e^{i \phi}\right)$ is determined completely by the behavior of $f\left(r e^{i \psi}\right)$ on the half-line $\psi=\pi-\phi$. As a result, there is no relation between the magnitudes of $\int_{0}^{\infty}\left|f\left(r e^{i \phi}\right)\right|^{2} d r$ and $\int_{0}^{\infty}\left|U f\left(r e^{i \phi}\right)\right|^{2} d r$.

In the next section, we define an operator class $\mathfrak{A}$ which does not have this undesirable feature. In particular, we introduce an $\alpha$-norm so as to make $\mathfrak{A}$ a Banach algebra. The $\alpha$-norm is investigated in some detail in $\$ 4.3$. This prepares for $\$ 4.4$, where it is proved that $\mathfrak{R}$ is an ideal in $\mathfrak{A}$.

4.2. The Banach algebra $\mathfrak{A}$.

Definition 4.1. The class of all linear operators $A$ mapping $\mathfrak{S}^{2}$ into $\mathfrak{S}^{2}$ such that

$$
\alpha(A, \phi)=\sup _{f \in \mathfrak{\Phi}^{2}}\left[\int_{0}^{\infty}\left|A f\left(r e^{i \phi}\right)\right|^{2} d r\right]^{1 / 2}\left[\int_{0}^{\infty}\left|f\left(r e^{i \phi}\right)\right|^{2} d r\right]^{-1 / 2}
$$

exists and is bounded, uniformly in $\phi$ for $0<\phi<\pi$, is denoted by $\mathfrak{A}$.

When studying the class $\mathfrak{A}$, it is convenient to use the following notation.

Definition 4.2. Let $f$ and $g$ be any two functions in $\mathfrak{S}^{2}$, and let their Mellin transforms be $f$ and $g$. Then the symbols $(f, g)_{\phi},(f, g)_{\phi},\|f\|_{\phi},\|f\|_{\phi}$ stand for

$$
\begin{array}{ll}
(f, g)_{\phi}=\int_{0}^{\infty} f\left(r e^{i \phi}\right) \bar{g}\left(r e^{i \phi}\right) d r, & \|f\|_{\phi}=\left[(f, f)_{\phi}\right]^{1 / 2}, \\
(f, g)_{\phi}=\int_{-\infty}^{\infty} e^{2 \phi t} f(t) \bar{g}(t) d t, & \|f\|_{\phi}=\left[(f, f)_{\phi}\right]^{1 / 2} .
\end{array}
$$

It follows from general formulas for Mellin transforms that

$$
(f, g)_{\phi}=(\boldsymbol{f}, \boldsymbol{g})_{\phi} .
$$

LEMMA 4.3. If $A$ is an operator in $\mathfrak{A}$, the quantity

$$
\alpha(A, \phi)=\sup _{f \in \mathfrak{g}^{2}}\|A f\|_{\phi} /\|f\|_{\phi}
$$

exists and is bounded, uniformly in $\phi$ for $0 \leqq \phi \leqq \pi$.

Proof. The $0<\phi<\pi$ part of the assertion is true by assumption. Also, given any $f$ in $\mathfrak{G}^{2}$, it is implied by Definition 4.1 that $A f$ is in $\mathfrak{H}^{2}$. Thus, there are boundary functions $A f(r)$ and $A f\left(r e^{i \pi}\right)$, as in equation (2.11). More specifically,

$$
\lim _{\phi \rightarrow 0}\|A f\|_{\phi} /\|f\|_{\phi}=\|A f\|_{0} /\|f\|_{0} \text {. }
$$

Now suppose that the $\phi=0$ part of the assertion is false. Given any large positive $N$, there would be an $f$ in $\mathfrak{S}^{2}$ such that

$$
\|A f\|_{0}>N\|f\|_{0}
$$


This would imply that we could make $\|A f\|_{\phi} /\|f\|_{\phi}$ as large as we please by first choosing $f$ in a suitable way, next choosing $\phi$ sufficiently close to 0 . But this violates the assumption that $A$ is in $\mathfrak{A}$. Thus, the $\phi=0$ part of the lemma is true, and similarly for the $\phi=\pi$ part. This proves the lemma.

Definition 4.4. Let $A$ be an operator in $\mathfrak{A}$. Then $\alpha(A)$ is defined by

$$
\alpha(A)=\sup _{0<\phi<\pi} \alpha(A, \phi) .
$$

Actually, it is shown in Theorem 4.10 that $\log \alpha(A, \phi)$ is a convex function of $\phi$. Hence

$$
\alpha(A)=\max [\alpha(A, 0), \alpha(A, \pi)] .
$$

It is easily seen that $\alpha(A)$ is a norm for operators $A$ in $\mathfrak{A}$, making $\mathfrak{A}$ a normed algebra. We proceed to show that $\mathfrak{A}$ is a Banach algebra.

LEMMA 4.5. If $A$ is in $\mathfrak{A}$, it is a bounded operator on $\mathfrak{S}^{2}$, the norm $\|A\|$ satisfying

$$
\|A\| \leqq \alpha(A) \text {. }
$$

Proof. Given an operator $A$ in $\mathfrak{A}$ and a function $f$ in $\mathscr{S}^{2}$, it follows from the proof of Lemma 4.3 that there exist quantities $\|A f\|_{\phi}$ with $\phi=0, \pi$. In fact, equation (4.12) implies that

$$
\|A f\|_{0} \leqq \alpha(A)\|f\|_{0},
$$

and similarly for $\phi=\pi$. Since

$$
\|A\|=\sup _{f \in \mathfrak{S}^{2}}\left(\|A f\|_{0}^{2}+\|A f\|_{\pi}^{2}\right)^{1 / 2}\left(\|f\|_{0}^{2}+\|f\|_{\pi}^{2}\right)^{-1 / 2},
$$

the desired relation (4.16) follows.

THEOREM 4.6. The class $\mathfrak{A}$ is complete in the $\alpha$-norm.

Proof. Let $\left\{A_{n}\right\}(n=1,2, \ldots)$ be a sequence of operators in $\mathfrak{A}$ such that $\alpha\left(A_{n}-A_{m}\right)$ tends to 0 as $n$ and $m$ tend to $\infty$. It follows from the triangle inequality for the $\alpha$-norm that $\left\{\alpha\left(A_{n}\right)\right\}$ is a Cauchy sequence and, hence, tends to a limit. Let this be denoted by $\alpha$.

Owing to equation (4.16), the quantity $\left\|A_{n}-A_{m}\right\|$ tends to 0 . Hence, there is a bounded linear operator $A$ on $\mathscr{S}^{2}$ such that $\left\|A_{n}-A\right\|$ tends to 0 . We want to show that $A$ belongs to $\mathfrak{X}$, with $\alpha\left(A_{n}-A\right)$ tending to 0 .

By virtue of equation (2.36),

$$
\|A f\|_{\phi} \leqq\left\|\left(A_{n}-A\right) f\right\|_{\phi}+\left\|A_{n} f\right\|_{\phi} \leqq\left\|A_{n}-A\right\|\|f\|+\alpha\left(A_{n}\right)\|f\|_{\phi} .
$$

Letting $n$ tend to $\infty$ gives

$$
\|A f\|_{\phi} \leqq \alpha\|f\|_{\phi}
$$

Hence, $A$ belongs to $\mathfrak{A}$, with $\alpha(A) \leqq \alpha$. 
It remains to show that $\alpha\left(A_{n}-A\right)$ tends to 0 , this implying, in particular, that $\alpha(A)=\alpha$. To complete this part of the proof, observe that $\left\{\alpha\left(A_{n}-A\right)\right\}$ is a Cauchy sequence, again by the triangle inequality for the $\alpha$-norm. Let the limit of the sequence be $\beta$, and suppose that $\beta>0$. Choose $M$ so large that $\alpha\left(A_{n}-A_{m}\right)<\beta / 4$ for $n, m>M$. By the definition of $\beta$, there is an $N$, which we may choose to be larger than $M$, such that, given any fixed $n>N$, there exists a function $f_{n}\left(r e^{i \phi}\right) \not \equiv 0$ in $\mathfrak{S}^{2}$, and an angle $\phi(n)$ satisfying

$$
\left\|\left(A_{n}-A\right) f_{n}\right\|_{\phi(n)} \geqq \frac{1}{2} \beta\left\|f_{n}\right\|_{\phi(n)} .
$$

For $n, m>N$, this gives

$$
\begin{aligned}
\left\|\left(A_{m}-A\right) f_{n}\right\|_{\phi(n)} & \geqq\left\|\left(A_{n}-A\right) f_{n}\right\|_{\phi(n)}-\left\|\left(A_{n}-A_{m}\right) f_{n}\right\|_{\phi(n)} \\
& \geqq \frac{1}{2} \beta\left\|f_{n}\right\|_{\phi(n)}-\alpha\left(A_{n}-A_{m}\right)\left\|f_{n}\right\|_{\phi(n)} \geqq \frac{1}{4} \beta\left\|f_{n}\right\|_{\phi(n)} .
\end{aligned}
$$

At the same time, equation (2.36) implies that

$$
\left\|\left(A_{m}-A\right) f_{n}\right\|_{\phi(n)} \leqq\left\|A_{m}-A\right\|\left\|f_{n}\right\| .
$$

Combining equations (4.22) and (4.23), keeping $n$ fixed, and letting $m$ tend to $\infty$ yields

$$
\frac{1}{4} \beta\left\|f_{n}\right\|_{\phi(n)}=0 .
$$

If $\beta \neq 0$, this requires that $f_{n}\left(r e^{i \phi(n)}\right)=0$. Since $f_{n}$ is analytic, it follows that $f_{n} \equiv 0$. This is a contradiction, hence $\beta=0$. Thus $\alpha\left(A_{n}-A\right)$ tends to 0 as $n$ tends to $\infty$, which proves the theorem.

COROLlaRy 4.7. The class $\mathfrak{A}$ is a Banach algebra under the $\alpha$-norm.

4.3. The $\alpha$-norm. If we are given a function $f$ in $\mathfrak{S}^{2}$ and we keep $\phi$ fixed, we have a function $f\left(r e^{i \phi}\right)$ in $\mathfrak{Q}^{2}$, for each fixed $\phi$. Similarly, if $A$ is an operator in $\mathfrak{A}$, it is shown below that it is associated with an operator $A(\phi)$ on $\mathfrak{Q}^{2}$, for each fixed $\phi$. To discuss this point, it is convenient to adopt the following notation.

Definition 4.8. If $g$ is an element of $\mathfrak{Q}^{2}$ and $B$ is a bounded linear operator on $\mathfrak{L}^{\mathbf{2}}$, their $\mathfrak{L}^{\mathbf{2}}$-norms are denoted by $\lambda(g)$ and $\lambda(B)$, respectively. The $\mathfrak{Z}^{2}$-norms of the Mellin transforms $g$ and $\boldsymbol{B}$ are denoted by $\lambda(\boldsymbol{g})$ and $\lambda(\boldsymbol{B})$.

LEMMA 4.9. If $A$ is in $\mathfrak{A}$, it is associated with a family of bounded linear operators $A(\phi)$ mapping the space $\mathfrak{Q}^{2}$ into $\mathfrak{Q}^{2}$ and satisfying

$$
\lambda[A(\phi)]=\alpha(A, \phi) .
$$

Here $0 \leqq \phi \leqq \pi$. For every $f$ in $\mathfrak{S}^{2}$,

$$
A(\phi) f\left(r e^{i \phi}\right)=A f\left(r e^{i \phi}\right),
$$

the function $f$ on the left also being regarded as an element of $\mathfrak{Q}^{2}$.

Proof. Let $A$ be fixed in $\mathfrak{A}$, let $f$ run through $\mathfrak{S}^{2}$, but consider $f\left(r e^{i \phi}\right)$ and $A f\left(r e^{i \phi}\right)$ only for some fixed $\phi$ in $0 \leqq \phi \leqq \pi$. According to Theorem $2.17, f\left(r e^{i \phi}\right)$ runs through 
a set which is dense in $\mathfrak{L}^{2}$. Let this set be denoted by $\mathfrak{D}(\phi)$ and define $A(\phi)$ by equation (4.26) for all $f$ in $\mathfrak{D}(\phi)$. Since $A$ is in $\mathfrak{A}$ by assumption, $A(\phi)$ thus defined is a bounded linear operator with domain $\mathfrak{D}(\phi)$, its norm being $\alpha(A, \phi)$. Since $\mathfrak{D}(\phi)$ is dense in $\mathfrak{L}^{2}$, the operator $A(\phi)$ on $\mathfrak{D}(\phi)$ has a unique closure with domain $\mathfrak{L}^{2}$. This closure we also denote by $A(\phi)$. It has all the properties required by the lemma.

Upon taking Mellin transforms, $\mathfrak{S}^{2}$ goes into $\mathfrak{\mathscr { Y }}^{2}$, the space $\mathfrak{Q}^{2}$ goes into $\mathfrak{Q}^{2}$, and $\mathfrak{D}(\phi)$ goes into a set $\mathfrak{D}(\phi)$ which is dense in $\mathfrak{2}^{2}$. It follows from Remark 2.10 that a function $g(t)$ is in $\mathfrak{D}(\phi)$ if and only if $g(t) \exp (-\phi t)$ and $g(t) \exp (\pi t-\phi t)$ are in $\mathbf{2}^{2}$.

Denoting the Mellin transform by $\Gamma$ and using equation (2.29) gives

$$
\Gamma A f\left(r e^{i \phi}\right)=A f(t, \phi)=e^{\phi t-i \phi / 2} A f(t),
$$

and so

$$
A f(t, \phi)=e^{\phi t-i \phi / 2} A e^{-\phi t+i \phi / 2} f(t, \phi)
$$

for every $f(t, \phi)$ in $\mathfrak{D}(\phi)$ and every $A$ in $\mathfrak{B}$.

Now let $A$ be in $\mathfrak{A}$. Consider the restriction of $A(\phi)$ to $\mathscr{D}(\phi)$ and denote the Mellin transform of this by $A(\phi)$. The operator $A(\phi)$ thus defined may be used instead of $\boldsymbol{A}$ on the left in equation (4.28). On the right, one would then replace $A$ by $A(0)$. The restriction of $A(\phi)$ to $\mathscr{D}(\phi)$ can be extended to all of $\mathcal{2}^{2}$ in the same way as $A(\phi)$ was extended to $\mathfrak{Q}^{2}$. This yields

$$
\alpha(A, \phi)=\lambda[A(\phi)]=\sup _{\boldsymbol{g} \in \mathfrak{R}^{2}} \lambda[A(\phi) g] / \lambda(\boldsymbol{g}) .
$$

Observe, however, that $\alpha(A, \phi)$ can also be obtained by taking the sup for $g$ running only through some convenient set contained in all domains $\mathcal{D}(\phi)$. This idea is used in the proof of the following theorem.

THEOREM 4.10. For any fixed operator $A$ in $\mathfrak{A}$, the function $\log \alpha(A, \phi)$ is a convex function of $\phi$ in $0 \leqq \phi \leqq \pi$.

Proof. Choose some $\phi$ in $0 \leqq \phi \leqq \pi$. The quantity $\alpha(A, \phi)$ can be found by letting $g$ in equation (4.29) run through the set $\boldsymbol{\epsilon}_{0}$ consisting of all bounded functions which vanish outside bounded intervals. If $g$ is in $\boldsymbol{E}_{0}$, it is in $\mathcal{D}(\phi)$. Hence $e^{\phi t} \boldsymbol{A} e^{-\phi t} \boldsymbol{g}(t)$ is in $\mathfrak{D}(\phi)$, by equation (4.28). This is the same as saying that $A e^{-\phi t} g(t)$ and $\boldsymbol{e}^{\pi t} \boldsymbol{A} \boldsymbol{e}^{-\phi t} \boldsymbol{g}(t)$ are in $\mathbf{2}^{2}$.

Now let $\phi$ take complex values in a strip,

$$
\phi=\sigma+i \tau \quad(0 \leqq \sigma \leqq \pi,-\infty<\tau<\infty),
$$

and consider the expression

$$
N(\phi)=\int_{-\infty}^{\infty}\left[e^{\phi t} A e^{-\phi t} g(t)\right][\overline{\exp (\phi t) A \exp (-\phi t) g(t)}] d t,
$$

keeping $A$ fixed in $\mathfrak{A}$ and $g$ fixed in $\mathfrak{C}_{0}$. Straightforward analysis shows that $N(\phi)$ is analytic in the interior of the strip and continuous on the lines $\sigma=0$ and $\sigma=\pi$. 
For $\sigma=0$, it follows with equation (4.29) that

$$
|N(i \tau)| \leqq \lambda\left[A(0) e^{-i \tau t} g(t)\right] \lambda\left[A(0) e^{i \tau t} g(t)\right] \leqq[\alpha(A, 0) \lambda(g)]^{2},
$$

and similarly for $\sigma=\pi$. The line analogue of Hadamard's three-circle theorem $[13$, p. 93] now says that, for $0<\sigma<\pi$,

$$
|N(\phi)| \leqq[\alpha(A, 0)]^{2-2 \phi / \pi}[\alpha(A, \pi)]^{2 \phi / \pi}[\lambda(g)]^{2} .
$$

If $\phi$ is real, $N(\phi)$ is nothing but $\{\lambda[A(\phi) g]\}^{2}$. Dividing by $[\lambda(g)]^{2}$ and taking the sup for $g$ running through $\mathfrak{\Xi}_{0}$ thus gives

$$
\alpha(A, \phi) \leqq[\alpha(A, 0)]^{1-\phi / \pi}[\alpha(A, \pi)]^{\phi / \pi} .
$$

This shows that $\log \alpha(A, \phi)$ is convex and thereby proves the theorem. The relation (4.15) has now also been justified.

REMARK 4.11. The equality sign occurs in equation (4.34) if $A$ is a constant multiple of the identity operator. It also occurs if

$$
A f\left(r e^{i \phi}\right)=\left(r e^{i \phi}\right)^{i u} f\left(r e^{i \phi}\right), \quad A f(t, \phi)=e^{\phi t-i \phi / 2} f(t+u),
$$

with some real $u$. In this case, $\alpha(A, \phi)=e^{-\phi u}$.

The proof of Theorem 4.10 leads to an alternative characterization of the class $\mathfrak{A}$, which we give for future reference.

THEOREM 4.12. The class $\mathfrak{A}$ is the class of all linear operators $A$ on $\mathfrak{S}^{2}$ for which there exist numbers $\alpha(A)$ such that

$$
\|A f\|_{0} \leqq \alpha(A)\|f\|_{0}, \quad\|A f\|_{\pi} \leqq \alpha(A)\|f\|_{\pi}
$$

for every $f$ in $\mathfrak{S}^{2}$.

Proof. If $A$ is in $\mathfrak{A}$, it is obvious from Definitions 4.1 and 4.4, combined with Lemma 4.3, that a number $\alpha(A)$ satisfying equation (4.36) must exist.

Now suppose that we are given equation (4.36). It must be shown that $A$ is in $\mathfrak{A}$. The data permit us to construct operators $A(0)$ and $A(\pi)$, as in Lemma 4.9. Their $\lambda$-norms do not exceed $\alpha(A)$. For any $g$ in $\boldsymbol{\xi}_{0}$, equation (4.31) defines an analytic function $N(\phi)$. Its boundary values $N(i \tau)$ and $N(\pi+i \tau)$ satisfy inequalities of the form (4.32). This gives an inequality of the form (4.33) for $N(\phi)$. It follows that $e^{\phi t} A e^{-\phi t}$ is a bounded operator on $\boldsymbol{\epsilon}_{0}$ whose norm does not exceed $\alpha(A)$. Since $\boldsymbol{\epsilon}_{0}$ is dense in $\mathbf{2}^{2}$, the operator $e^{\phi t} A e^{-\phi t}$ can be extended to all of $\mathbf{Z}^{2}$ without increasing its norm. Let the extension be denoted by $A(\phi)$. The restriction of $A(\phi)$ to $\mathscr{D}(\phi)$ is all that is required to find $A f(t, \phi)$, next $A f\left(r e^{i \phi}\right)$, for any $f$ in $\mathscr{S}^{2}$. Since the $\lambda$-norm of $e^{\phi t} A e^{-\phi t}$ does not exceed $\alpha(A)$,

$$
\lambda\left[e^{\phi t} A e^{-\phi t} f(t, \phi)\right] \leqq \alpha(A) \lambda[f(t, \phi)]
$$

which is the same as

$$
\|A f\|_{\phi} \leqq \alpha(A)\|f\|_{\phi} .
$$

This shows that $A$ is in $\mathfrak{U}$ and completes the proof. 
4.4. Operators in the algebra $\mathfrak{A}$. It is proved in Theorem 4.14 that $\mathfrak{A}$ is an ideal in $\mathfrak{A}$. It is pointed out in Remark 4.18 that there are many operators of rank 1 which do not belong to $\mathfrak{A}$. Thus, $\mathfrak{A}$ is small in a sense, yet it is found in Theorem 4.19 that $\mathfrak{A}$ is dense in $\mathfrak{B}$, in the strong topology on $\mathfrak{S}^{2}$. If one chooses a fixed $\phi$ and considers all operators $A(\phi)$ associated with operators $A$ in $\mathfrak{A}$, one obtains a set which is dense in the algebra of all bounded linear operators on $\mathfrak{Q}^{2}$, by Theorem 4.22 .

LEMmA 4.13. If $K$ is an operator in $\mathfrak{R}$, then it is in $\mathfrak{A}$, and

$$
\alpha(K) \leqq \kappa(K) .
$$

Proof. Let $K$ be in $\Re$ and let its kernel be $K\left(r, r^{\prime}, \phi\right)$. By Schwarz's inequality and equation (3.23),

$$
\|K f\|_{\phi} \leqq\left[\int_{0}^{\infty} \int_{0}^{\infty}\left|K\left(r, r^{\prime}, \phi\right)\right|^{2} d r d r^{\prime}\right]^{1 / 2}\|f\|_{\phi} \leqq \kappa(K)\|f\|_{\phi}
$$

for every $f$ in $\mathfrak{S}^{2}$. From this the lemma follows immediately.

THEOREM 4.14. Let $K$ and $A$ be operators in $\mathfrak{\Re}$ and $\mathfrak{A}$, respectively. Then $A K$ and $K A$ are in $\Re$, with

$$
\kappa(A K) \leqq \alpha(A) \kappa(K), \quad \kappa(K A) \leqq \alpha(A) \kappa(K) .
$$

Proof. Since $K$ and $A$ are in $\mathfrak{A}$, so is $A K$. For fixed $\phi$, Lemma 4.9 says that there are extensions $K(\phi), A(\phi)$, and $(A K)(\phi)$ mapping $\mathfrak{L}^{2}$ into $\mathfrak{Q}^{2}$. In fact, it is easy to see that $K(\phi)$ is an integral operator having the same kernel as $K$. Let this be $K\left(r, r^{\prime}, \phi\right)$. Since this is a function in $\mathfrak{Q}^{2}$, it follows that $K(\phi)$ is in the Schmidt class on $\mathfrak{Q}^{2}$. Its Schmidt norm on $\mathfrak{Q}^{2}$ is equal to

$$
\left[\int_{0}^{\infty} \int_{0}^{\infty}\left|K\left(r, r^{\prime}, \phi\right)\right|^{2} d r d r^{\prime}\right]^{1 / 2}
$$

Furthermore, $A(\phi)$ is bounded on $\mathfrak{L}^{2}$ and has $\lambda$-norm $\alpha(A, \phi)$. From this it follows that $A(\phi) K(\phi)$ is in the Schmidt class on $\mathfrak{Q}^{2}$ and has a Schmidt norm not exceeding

$$
\alpha(A, \phi)\left[\int_{0}^{\infty} \int_{0}^{\infty}\left|K\left(r, r^{\prime}, \phi\right)\right|^{2} d r d r^{\prime}\right]^{1 / 2} \text {. }
$$

More specifically, it follows that $A(\phi) K(\phi)$ is an integral operator with an $\mathfrak{2}^{2}$ kernel whose norm does not exceed the quantity (4.43). But $A(\phi) K(\phi)$ is the same operator as $(A K)(\phi)$. Thus, there is a $\operatorname{kernel}(A K)\left(r, r^{\prime}, \phi\right)$, say, such that

$$
\left[\int_{0}^{\infty} \int_{0}^{\infty}\left|(A K)\left(r, r^{\prime}, \phi\right)\right|^{2} d r d r^{\prime}\right]^{1 / 2} \leqq \alpha(A, \phi)\left[\int_{0}^{\infty} \int_{0}^{\infty}\left|K\left(r, r^{\prime}, \phi\right)\right|^{2} d r d r^{\prime}\right]^{1 / 2}
$$

Now observe that the operator $A K$ on $\mathfrak{S}^{2}$ is obtained by first restricting $(A K)(\phi)$ to the set $\mathfrak{D}(\phi)$, next letting $\phi$ run from 0 to $\pi$. This gives

$$
A K f\left(r e^{i \phi}\right)=\int_{0}^{\infty}(A K)\left(r, r^{\prime}, \phi\right) f\left(r^{\prime} e^{i \phi}\right) e^{i \phi} d r^{\prime}
$$


for all $f$ in $\mathfrak{S}^{2}$. Since the right-hand side of equation (4.44) is bounded uniformly in $\phi$ for $0<\phi<\pi$, by assumption, it follows that $A K$ is in $\Re$. The definitions of the $\kappa$ - and $\alpha$-norms, equations (3.22) and (4.14), now give the first relation (4.41). There is a similar argument for the operator $K A$ and the second relation (4.41). This completes the proof of the theorem.

COROllary 4.15. The class $\mathfrak{\Re}$ is an ideal in the Banach algebra $\mathfrak{A}$.

If $S$ is an operator in the Schmidt class $\subseteq$ having Schmidt norm $\sigma(S)$, and if $V$ is unitary, then it is well known [6, p. 30] that both $V S$ and $S V$ are in $\subseteq$ and have Schmidt norms $\sigma(S)$. The analogous property of the class $\Re$ is expressed by the following theorem.

THEOREM 4.16. Let $K$ be an operator in $\Re$, let $V$ be a bounded linear operator mapping $\mathfrak{S}^{2}$ onto $\mathfrak{S}^{2}$, and suppose that

$$
(V f, V g)_{0}=(f, g)_{0}, \quad(V f, V g)_{\pi}=(f, g)_{\pi}
$$

for every $f$ and $g$ in $\mathfrak{S}^{2}$. Then $V K$ and $K V$ are operators in $\Re$ satisfying

$$
\kappa(V K)=\kappa(K V)=\kappa(K) \text {. }
$$

Proof. The operator $V$ is in $\mathfrak{A}$ by Theorem 4.12. Hence, there are operators $V(0)$ and $V(\pi)$ defined along the lines of Lemma 4.9. Owing to equation (4.46), these have unit norm.

Now let $f$ run through $\mathfrak{S}^{2}$. This makes $f(r)$ run through $\mathfrak{D}(0)$. Since the range of $V$ is equal to $\mathfrak{S}^{2}$, by assumption, $V f(r)$ also runs through $\mathfrak{D}(0)$. The operator $V(0)$ with domain $\mathfrak{L}^{2}$ is obtained by extending $V$ from $\mathfrak{D}(0)$ to all of $\mathfrak{L}^{2}$. With the help of equation (4.46), it is easy to prove that the range of $V(0)$ is all of $\mathfrak{R}^{2}$. Hence, $V(0)$ is unitary on $\mathfrak{Q}^{2}$. And similarly for $V(\pi)$.

Now consider the operator $K(0)$, which is in the Schmidt class on $\mathfrak{2}^{2}$. As in the proof of Theorem 4.14, $(V K)(0)$ is also in the Schmidt class on $\mathfrak{R}^{2}$. Since $V(0)$ is unitary, $K(0)$ and $(V K)(0)$ have the same Schmidt norm on $\mathfrak{2}^{2}$. Let the kernels of these operators be $K\left(r, r^{\prime}, 0\right)$ and $(V K)\left(r, r^{\prime}, 0\right)$, respectively. On $\mathfrak{R}^{2}$, the Schmidt norm is the norm of the kernel of the operator in question. Hence

$$
\int_{0}^{\infty} \int_{0}^{\infty}\left|(V K)\left(r, r^{\prime}, 0\right)\right|^{2} d r d r^{\prime}=\int_{0}^{\infty} \int_{0}^{\infty}\left|K\left(r, r^{\prime}, 0\right)\right|^{2} d r d r^{\prime}
$$

and similarly for $\phi=\pi$. From this it follows with equation (3.22) that $\kappa(V K)=\kappa(K)$. A similar argument shows that $\kappa(K V)=\kappa(K)$.

REMARK 4.17. An operator $V$ mapping $\mathfrak{S}^{2}$ onto $\mathfrak{S}^{2}$ and satisfying equation (4.46) is unitary on $\mathfrak{S}^{2}$. The operator $U$ defined by equation (4.3) is unitary but does not belong to $\mathfrak{A}$ and does not satisfy equation (4.46).

REMARK 4.18. Consider the operator $S$ corresponding to the kernel $S\left(t, t^{\prime}\right)$ given in equation (3.35). This is an operator of rank 1. By equation (3.28), it acts according to

$$
S f(t, \phi)=\int_{-\infty}^{\infty} e^{\phi t} a(t) \bar{b}\left(t^{\prime}\right)\left(1+\exp \left(2 \pi t^{\prime}\right)\right) \exp \left(-\phi t^{\prime}\right) f\left(t^{\prime}, \phi\right) d t^{\prime}
$$


where $f(t, \phi)$ takes values in $\mathcal{D}(\phi)$. The integral operator on the right can be extended to all of $\mathbf{2}^{\mathbf{2}}$ if and only if

$$
\boldsymbol{b}(t)\left(1+e^{2 \pi t}\right) e^{-\phi t} \in \mathbf{Q}^{2} .
$$

In order that $S$ be in $\mathfrak{A}$, Lemma 4.9 thus requires that $\boldsymbol{b}(t) e^{2 \pi t}$ and $\boldsymbol{b}(t) e^{-\pi t}$ be in $\mathbf{2}^{2}$, a condition which is also sufficient for $S$ to be in $\mathfrak{A}$. From this it follows that there are many examples of operators of rank 1 which are not in $\mathfrak{A}$. In fact, when combined with the proof of Theorem 3.11, the present remark implies that an operator of rank 1 is in $\mathfrak{A}$ if and only if it is in $\Re$.

We complete this section with results analogous to Theorems 3.12 and 2.16.

THEOREM 4.19. Given a bounded linear operator $B$ on $\mathfrak{S}^{2}$, there is a sequence of operators $A_{T}$ in $\mathfrak{A}$ such that

$$
\lim _{T \rightarrow \infty}\left\|\left(B-A_{T}\right) f\right\|=0
$$

for every $f$ in $\mathfrak{S}^{2}$.

Proof. For any $T>0$, define the operator $\boldsymbol{P}_{T}$ and its inverse Mellin transform $\boldsymbol{P}_{T}$ by the requirement that

$$
\begin{aligned}
\boldsymbol{P}_{T} f(t, \phi) & =f(t, \phi) & & (-T<t<T), \\
& =0 & & (|t|>T),
\end{aligned}
$$

for every $\boldsymbol{f}$ in $\mathfrak{\mathcal { Q }}^{2}$. Given an operator $B$ with norm $\|B\|$, it follows with equation (2.36) that

$$
\begin{aligned}
\left\|B P_{T} f\right\|_{\phi} & \leqq\|B\|\left\|P_{T} f\right\|=\|B\|\left[\int_{-T}^{T}\left(1+e^{2 \pi t}\right)|f(t)|^{2} d t\right]^{1 / 2} \\
& \leqq\|B\| e^{\pi T}\left[2 \int_{-T}^{T} e^{2 \phi t}|f(t)|^{2} d t\right]^{1 / 2} \leqq 2^{1 / 2}\|B\| e^{\pi T}\|f\|_{\phi^{*}}
\end{aligned}
$$

Hence, $B P_{T}$ is in $\mathfrak{A}$. Choosing $A_{T}=B P_{T}$ yields an operator $A_{T}$ in $\mathfrak{A}$ which tends to $B$ strongly as $T$ tends to $\infty$. This proves the theorem.

REMARK 4.20. Consider the operator $U$ defined by equation (4.3). There is no operator $A$ in $\mathfrak{A}$ such that $\|U-A\|<1$.

Proof. If $A$ is an operator in $\mathfrak{A}$ and if $f$ is any function in $\mathfrak{S}^{2}$, we have

$$
\|U f\|_{0} \leqq\|A f\|_{0}+\|U-A\|\|f\| \leqq \alpha(A)\|f\|_{0}+\|U-A\|\|f\| .
$$

This is the same statement as

$$
\begin{aligned}
& {\left[\int_{-\infty}^{\infty} e^{2 \pi t}|f(t)|^{2} d t\right]^{1 / 2}} \\
& \quad \leqq \alpha(A)\left[\int_{-\infty}^{\infty}|f(t)|^{2} d t\right]^{1 / 2}+\|U-A\|\left[\int_{-\infty}^{\infty}\left(1+e^{2 \pi t}\right)|f(t)|^{2} d t\right]^{1 / 2} .
\end{aligned}
$$

Now if $\|U-A\|<1$, equation (4.55) is violated whenever the support of $f$ is confined to $t$-values that are sufficiently large. This proves the assertion. 
COROLlaRY 4.21. The operator $U$ defined by equation (4.3) cannot be approximated uniformly by operators in $\mathfrak{A}$.

THEOREM 4.22. Given a bounded linear operator $B$ on $\mathfrak{L}^{2}$ and a fixed angle $\psi$ in $0 \leqq \psi \leqq \pi$, there is a sequence of operators $A_{T}$ in $\mathfrak{A}$ such that

$$
\lim _{T \rightarrow \infty} \lambda\left[B g-A_{T}(\psi) g\right]=0
$$

for every $g$ in $\mathfrak{Q}^{2}$.

Proof. Let $A_{T}$ and its inverse Mellin transform $A_{T}$ be defined by

$$
\boldsymbol{A}_{T} f(t, \phi)=\exp \left(\phi t-\frac{1}{2} i \phi-\psi t+\frac{1}{2} i \psi\right) \boldsymbol{P}_{T} \boldsymbol{B P}_{T} f(t, \psi),
$$

where $f$ runs through $\tilde{\mathfrak{E}}^{2}$. This clearly gives an operator $A_{T}$ mapping $\mathfrak{S}^{2}$ into $\mathfrak{S}^{2}$. It is easy to prove that

$$
\left\|A_{T} f\right\|_{\phi} \leqq e^{2 \pi T}\|B\|\|f\|_{\phi},
$$

hence that $A_{T}$ is in $\mathfrak{A}$. The extension $A_{T}(\psi)$ on $\mathfrak{R}^{2}$ is equal to $P_{T} B P_{T}$. Since this is an operator which tends to $B$ strongly as $T$ tends to $\infty$, the theorem follows.

REMARK 4.23. Consider the operator $B$ on $\mathfrak{Q}^{2}$ whose Mellin transform $B$ on $\mathfrak{Q}^{2}$ acts according to $\boldsymbol{B g}(t)=\boldsymbol{g}(-t)$. There is no operator $A$ in $\mathfrak{A}$ such that $\lambda[B-A(\psi)]$ $<1$ for some $\psi$ in $0 \leqq \psi \leqq \pi$.

Proof. If $A$ is any operator in $\mathfrak{A}$ and $g$ is in $\mathfrak{\mho}_{0}$, then

$$
\lambda[B \boldsymbol{g}-\boldsymbol{A}(\dot{\psi}) \boldsymbol{g}] \leqq \lambda[B-A(\psi)] \lambda(\boldsymbol{g}) .
$$

Now suppose that $\psi<\pi$ and choose a positive $\delta$ such that $\psi+\delta \leqq \pi$. Then

$$
\left[\int_{-\infty}^{T} e^{2 \delta t}|\operatorname{Bg}(t)|^{2} d t\right]^{1 / 2} \leqq \lambda\left[e^{\delta t} A(\psi) g\right]+e^{\delta T} \lambda[B-A(\psi)] \lambda(g) .
$$

Since $\boldsymbol{g}$ is in $\boldsymbol{\mho}_{0}$ by assumption, $\boldsymbol{g}$ can be regarded as a function $\boldsymbol{f}(t, \psi)$, where $\boldsymbol{f}$ is in $\mathfrak{\mathscr { Q }}^{2}$. This gives

$$
A(\psi) g(t)=A f(t, \psi),
$$

and the first term on the right in equation (4.60) is nothing but $\|A f\|_{\psi+\delta}$. If $A$ is in $\mathfrak{A}$, then $\|\boldsymbol{A f}\|_{\psi+\delta}$ does not exceed $\alpha(A)\|f\|_{\psi+\delta}$, which in turn is equal to $\alpha(A) \lambda\left(e^{\delta t} g\right)$. Written out explicitly, equation (4.60) thus gives

$$
\begin{aligned}
& {\left[\int_{-\infty}^{T} e^{2 \delta t}|g(-t)|^{2} d t\right]^{1 / 2}} \\
& \quad \leqq \alpha(A)\left[\int_{-\infty}^{\infty} e^{2 \delta t}|g(t)|^{2} d t\right]^{1 / 2}+e^{\delta T} \lambda[B-A(\psi)]\left[\int_{-\infty}^{\infty}|g(t)|^{2} d t\right]^{1 / 2}
\end{aligned}
$$

Now suppose that $\lambda[B-A(\psi)]<1$. The relation (4.62) is then violated if the support of $g$ is restricted to some interval $T_{0}<-t<T$, where $T_{0}$ and $T$ are positive and sufficiently large. This proves the assertion for the case $\psi<\pi$. To cover the 
case $\psi=\pi$, one can choose a negative $\delta$ and integrate from $-T$ to $\infty$ in equation (4.60).

COROLlaRY 4.24. In the case of the operator $B$ on $\mathfrak{Q}^{2}$ considered in Remark 4.23, there is no sequence of operators $A_{T}$ in $\mathfrak{A}$ such that, as $T$ tends to $\infty$, the sequence $A_{T}(\psi)$ tends to $B$, uniformly in the $\lambda$-norm, for some fixed $\psi$ in $0 \leqq \psi \leqq \pi$.

\section{Integral equations.}

5.1. Inhomogeneous Fredholm equations. Consider an integral equation of the form

$$
f\left(r e^{i \phi}\right)=g\left(r e^{i \phi}\right)+\gamma \int_{0}^{\infty} K\left(r, r^{\prime}, \phi\right) f\left(r^{\prime} e^{i \phi}\right) e^{i \phi} d r^{\prime}
$$

where $f$ and $g$ are in $\mathfrak{S}^{2}$, the function $K\left(r, r^{\prime}, \phi\right)$ is the kernel of an operator $K$ in $\Re$, and $\gamma$ is a complex number. Suppose that $g$ is known and that $f$ is to be found. Since $\mathfrak{R}$ is a subclass of the Schmidt class $\subseteq$ on $\mathfrak{S}^{2}$, equation (5.1) can be solved by a known generalization of the Fredholm theory of integral equations [8, pp. 96, 97]. Specifically, if $1 / \gamma$ is not an eigenvalue of $K$, there is a unique solution of the form

$$
f=g+\gamma \Delta(\gamma) g / \delta(\gamma) .
$$

For fixed $\gamma$, the quantity $\Delta(\gamma)$ is an operator in $\subseteq$, the quantity $\delta(\gamma)$ is a complex number. Both $\Delta(\gamma)$ and $\delta(\gamma)$ are entire functions of $\gamma$. The coefficients in the series expansions

$$
\Delta(\gamma)=\sum_{n=0}^{\infty} \Delta_{n} \gamma^{n}, \quad \delta(\gamma)=\sum_{n=0}^{\infty} \delta_{n} \gamma^{n}
$$

can be obtained from the recurrence relations

$$
\begin{aligned}
\Delta_{0} & =K, \quad \delta_{0}=1, & & \\
\Delta_{n+1} & =\delta_{n+1} K+K \Delta_{n} & & (n \geqq 0), \\
-(n+1) \delta_{n+1} & =\operatorname{trace}\left(\Delta_{n}-\delta_{n} K\right) & & (n \geqq 0) .
\end{aligned}
$$

The series for $\Delta(\gamma)$ converges in the Schmidt norm. The function $\delta(\gamma)$ vanishes at $\gamma=\gamma_{0}$ if and only if $1 / \gamma_{0}$ is an eigenvalue of $K[8, \mathrm{p} .100]$.

It follows from the second relation (5.4) by an induction argument that $\Delta_{n+1}$ is an operator in $\Re$ with kernel

$$
\Delta_{n+1}\left(r, r^{\prime}, \phi\right)=\delta_{n+1} K\left(r, r^{\prime}, \phi\right)+\int_{0}^{\infty} K\left(r, r^{\prime \prime}, \phi\right) \Delta_{n}\left(r^{\prime \prime}, r^{\prime}, \phi\right) e^{i \phi} d r^{\prime \prime} .
$$

As for the trace in equation (5.4), the trace class $\mathfrak{T}$ is the class of all operators $T$ which can be written as products of two operators in $\mathfrak{S}[6, p .37]$. If $T$ is in $\mathfrak{T}$, it has a polar decomposition of the form

$$
T f=\sum_{n} \tau_{n} a_{n}\left(f, b_{n}\right),
$$


where $\left\{a_{n}\right\}$ and $\left\{b_{n}\right\}$ are orthonormal sets, the quantities $\tau_{n}$ are positive numbers, and $\sum_{n} \tau_{n}$ converges [6, p. 41]. The trace of the operator (5.6) is given by [6, p. 37]

$$
\text { trace } T=\sum_{n}\left(T b_{n}, b_{n}\right) \text {. }
$$

In the special case of the space $\mathfrak{S}^{2}$, this leads to the following lemma.

LEMMA 5.1. Let $M$ be an operator of the form $M=K_{2} K_{1}$, where $K_{2}$ and $K_{1}$ are in $\Re$. Let $M\left(r, r^{\prime}, \phi\right)$ be the kernel of $M$ and let the Mellin transform $M\left(t, t^{\prime}\right)$ be defined as in equation (3.3). Then $M$ is in the trace class $\mathfrak{T}$, with

$$
\operatorname{trace} M=\int_{-\infty}^{\infty} M(t, t) d t=\int_{0}^{\infty} M(r, r, \phi) e^{i \phi} d r
$$

Proof. Since $\Re$ is a subclass of $\mathfrak{S}$, the product of two operators in $\Re$ belongs to $\mathfrak{T}$. Thus, $M$ is in $\mathfrak{T}$. Since the Mellin transform is a unitary mapping of $\mathfrak{Q}^{2}$ onto $\mathfrak{\mathscr { Q }}^{2}$, the trace of $M$ on $\mathscr{S}^{2}$ is equal to the trace of $M$ on $\mathscr{\mathcal { Q }}^{2}$.

The function $M\left(t, t^{\prime}\right)$ has a decomposition of the form

$$
M\left(t, t^{\prime}\right)=\sum_{n} \mu_{n} a_{n}(t) \bar{b}_{n}\left(t^{\prime}\right)\left(1+e^{2 \pi t^{\prime}}\right),
$$

where $\left\{\boldsymbol{a}_{n}\right\}$ and $\left\{\boldsymbol{b}_{n}\right\}$ are orthonormal sets in $\mathfrak{\mathscr { Q }}^{2}$, the quantities $\mu_{n}$ are positive numbers, and $\sum_{n} \mu_{n}$ converges. As in the proof of Theorem 3.10, it is convenient to consider

$$
N\left(t, t^{\prime}\right)=\left(1+e^{2 \pi t}\right)^{1 / 2} M\left(t, t^{\prime}\right)\left(1+e^{2 \pi t^{\prime}}\right)^{-1 / 2} .
$$

This is the kernel of an operator $N$ in the trace class on $\mathbf{2}^{2}$. Since $\left\{\left(1+e^{2 \pi t}\right)^{1 / 2} a_{n}\right\}$ and $\left\{\left(1+e^{2 \pi t}\right)^{1 / 2} b_{n}\right\}$ are orthonormal sets in $\mathbf{2}^{2}$, it follows from equation (5.7) that the trace of $N$ on $\mathfrak{Q}^{2}$ is equal to the trace of $M$ on $\mathfrak{\mathscr { Q }}^{2}$. But on $\mathcal{2}^{2}$, it is known that

$$
\operatorname{trace} N=\int_{-\infty}^{\infty} N(t, t) d t
$$

This justifies the first half of equation (5.8). To obtain the second half, it suffices to take $t^{\prime}=t$ in a relation of the form (3.3) and to integrate over $t$. The assumption that $M$ is a product $K_{2} K_{1}$ takes care of all convergence questions and yields equation (5.8) without difficulty. That the integral in equation (5.8) does not depend on $\phi$ is analogous to the observation made in Corollary 2.8. This proves the lemma.

COROllary 5.2. Combining the above lemma with equation (5.4) yields

$$
-(n+1) \delta_{n+1}=\int_{0}^{\infty}\left[\Delta_{n}(r, r, \phi)-\delta_{n} K(r, r, \phi)\right] e^{i \phi} d r \quad(n \geqq 0) .
$$

Since $\delta_{n+1}$ is the trace of a product of operators in $\Re$, it does not depend on $\phi$. 
It was already mentioned above that the series (5.3) for $\Delta(\gamma)$ converges in the Schmidt norm. In the case of equations with kernels in $\Re$ we have the following more powerful result.

LEMma 5.3. Let $K$ be an operator in $\mathfrak{\Re}$ and let quantities $\Delta_{n}$ and $\delta_{n}$ be defined according to equation (5.4). Then the series (5.3) for $\Delta(\gamma)$ converges in the $\kappa$-norm to an operator in $\AA$ which acts according to

$$
\Delta(\gamma) f\left(r e^{i \phi}\right)=\int_{0}^{\infty} \Delta\left(r, r^{\prime}, \phi ; \gamma\right) f\left(r^{\prime} e^{i \phi}\right) e^{i \phi} d r^{\prime},
$$

for every $f$ in $\mathfrak{S}^{2}$. Here $\Delta\left(r, r^{\prime}, \phi ; \gamma\right)$ is an element of the function class $\Re$.

Proof. If $\phi$ is kept fixed, the relation (5.1) can be regarded as an equation on $\mathfrak{i}^{2}$. In the notation of Lemma 4.9, the kernel on $\mathfrak{L}^{2}$ is $K(\phi)$. This is an operator in the Schmidt class on $\mathfrak{2}^{2}$, for every fixed $\phi$. To solve the equation on $\mathfrak{R}^{2}$, one needs quantities $\Delta(\gamma)$ and $\delta(\gamma)$, as in equation (5.2). These can be found from recurrence relations which are easily seen to take exactly the form (5.4). Now, from the theory on $\mathfrak{R}^{2}$ it is well known $[8$, p. 95] that

$$
\begin{array}{rl}
\int_{0}^{\infty} \int_{0}^{\infty}\left|\Delta_{n+1}\left(r, r^{\prime}, \phi\right)\right|^{2} & d r d r^{\prime} \\
& \leqq e(e / n)^{n}\left[\int_{0}^{\infty} \int_{0}^{\infty}\left|K\left(r, r^{\prime}, \phi\right)\right|^{2} d r d r^{\prime}\right]^{n+1} \quad(n \geqq 1) .
\end{array}
$$

On $\mathfrak{S}^{2}$, equation (3.22) therefore gives

$$
\kappa\left(\Delta_{n+1}\right) \leqq e^{1 / 2}(e / n)^{n / 2}[\kappa(K)]^{n+1} \quad(n \geqq 1) .
$$

From this it follows that the series for $\Delta(\gamma)$ converges in the $\kappa$-norm. Since $\Re$ is a Hilbert space, the limit $\Delta(\gamma)$ is in $\Re$. Hence, it is an integral operator according to equation (5.13). This proves the lemma.

The foregoing results permit the situation to be summarized as follows.

THEOREM 5.4. Let $K$ be an operator in $\AA$ and let $g$ be a function in $\mathfrak{S}^{2}$. Define quantities $\Delta_{n}$ and $\delta_{n}$ by equations (5.4), (5.5), and (5.12). The series (5.3) then converge for all complex $\gamma$, the series for $\Delta(\gamma)$ converging in the $\kappa-n o r m$ to an operator in $\Re$ which acts according to equation (5.13). The function $\delta(\gamma)$ vanishes at $\gamma=\gamma_{0}$ if and only if $1 / \gamma_{0}$ is an eigenvalue of $K$. If $\delta(\gamma) \neq 0$, the equation (5.1) has a unique solution $f$ in $\mathfrak{S}^{2}$ given by equation (5.2).

THEOREM 5.5. Let the data be as in Theorem 5.4, but restrict equation (5.1) to some fixed $\phi$ in $0 \leqq \phi \leqq \pi$ and regard the resulting relation as an equation on $\mathfrak{L}^{2}$ with kernel $K(\phi)$. The function $\delta(\gamma)$ vanishes at $\gamma=\gamma_{0}$ if and only if $1 / \gamma_{0}$ is an eigenvalue of $K(\phi)$. If $\delta(\gamma) \neq 0$, the equation on $\mathfrak{Q}^{2}$ has a unique solution which is the restriction to fixed $\phi$ of the solution on $\mathfrak{S g}^{2}$ considered in Theorem 5.4. 
Proof. This result follows from observations made in the proof of Lemma 5.3. Notice that, once the solution on $\mathfrak{L}^{2}$ is available for any particular fixed $\phi$, the solution on $\mathfrak{S E}^{2}$ can be obtained along the lines of Remark 2.11.

5.2. Homogeneous Fredholm equations. If $\delta(\gamma)$ vanishes for a particular $\gamma$, it is implied by Theorem 5.4 that the equation

$$
\gamma \int_{0}^{\infty} K\left(r, r^{\prime}, \phi\right) f\left(r^{\prime} e^{i \phi}\right) e^{i \phi} d r^{\prime}=f\left(r e^{i \phi}\right)
$$

has at least one solution in $\mathfrak{S}^{2}$. Obviously, this is also a solution in $\mathfrak{Q}^{2}$ of the equation restricted to fixed $\phi$. Let the number of linearly independent solutions in $\mathfrak{S}^{2}$ be denoted by $N$, and let $N(\phi)$ be the corresponding number on $\mathfrak{L}^{2}$. It is the purpose of the present section to show that $N=N(\phi)$, for $0 \leqq \phi \leqq \pi$. The proof uses properties of the adjoint operators $K^{*}$ and $K^{*}(\phi)$.

Lemma 5.6. Let $K$ be an operator in $\Re$ and let $K$ be its Mellin transform on $\mathfrak{\Phi}^{2}$. The adjoint operator $K^{*}$ on $\mathfrak{\Psi}^{2}$ acts according to

$$
\boldsymbol{K}^{*} \boldsymbol{f}(t, \phi)=\int_{-\infty}^{\infty} e^{\phi t}\left(1+e^{2 \pi t}\right)^{-1} \bar{K}\left(t^{\prime}, t\right)\left(1+\exp \left(2 \pi t^{\prime}\right)\right) \exp \left(-\phi t^{\prime}\right) f\left(t^{\prime}, \phi\right) d t^{\prime}
$$
for every $\boldsymbol{f}$ in $\mathfrak{\underline { \Psi }}^{2}$.

Proof. This follows from equation (3.21) and the definition of an adjoint operator.

Lemma 5.7. Let $K$ be an operator in $\Re$, and let $K$ be its Mellin transform. Choose some fixed $\phi$ in $0 \leqq \phi \leqq \pi$ and define the operator $K(\phi)$ by

$$
\boldsymbol{K}(\phi) \boldsymbol{f}(t, \phi)=\boldsymbol{K} \boldsymbol{f}(t, \phi),
$$

for every $f(t, \phi)$ in $\mathfrak{D}(\phi)$. If $K(\phi)$ is regarded as an operator on $\mathbf{2}^{2}$, its adjoint $\boldsymbol{K}^{*}(\phi)$ on $\mathbf{2}^{2}$ acts according to

$$
K^{*}(\phi) h(t)=\int_{-\infty}^{\infty} e^{-\phi t} \bar{K}\left(t^{\prime}, t\right) \exp \left(\phi t^{\prime}\right) h\left(t^{\prime}\right) d t^{\prime},
$$

for every $\boldsymbol{h}$ in $\mathbf{2}^{2}$.

Proof. This also follows from equation (3.21).

THEOREM 5.8. Let $K$ be an operator in $\Re$, choose some fixed $\phi$ in $0 \leqq \phi \leqq \pi$, and let $K(\phi)$ be the operator on $\mathfrak{Q}^{2}$ associated with $K$ in the sense of Lemma 4.9. Define the function $\delta(\gamma)$ as in Theorem 5.4. Suppose that $\delta(\gamma)=0$, so that $1 / \gamma$ is an eigenvalue of $K$ and $K(\phi)$. Let its multiplicities be denoted by $N$ and $N(\phi)$, respectively. Then $N=N(\phi)$, and each eigenvector of $K(\phi)$ is the restriction to fixed $\phi$ of an eigenvector of $K$.

Proof. The Mellin transform of equation (5.16) reads

$$
\gamma \int_{-\infty}^{\infty} e^{\phi t} K\left(t, t^{\prime}\right) \exp \left(-\phi t^{\prime}\right) f\left(t^{\prime}, \phi\right) d t^{\prime}=f(t, \phi) .
$$


We are assuming that this has $N$ linearly independent solutions in $\mathfrak{\mathfrak { E }}^{2}$. Since each solution in $\mathfrak{\mathscr { Q }}^{2}$ can be restricted to fixed $\phi$ to give a solution in $\mathbf{Q}^{2}$, it is obvious that $N(\phi) \geqq N$.

Now remember that, if $1 / \gamma$ is an eigenvalue of a Schmidt-class operator $S$, then $1 / \bar{\gamma}$ is an eigenvalue of $S^{*}$, the multiplicities of $1 / \gamma$ and $1 / \bar{\gamma}$ being equal [8, p. 103]. Thus, if equation (5.20) is regarded as an equation on $\mathbf{2}^{2}$, it follows with Lemma 5.7 that the equation

$$
\bar{\gamma} \int_{-\infty}^{\infty} e^{-\psi t} \bar{K}\left(t^{\prime}, t\right) \exp \left(\psi t^{\prime}\right) \boldsymbol{h}\left(t^{\prime}\right) d t^{\prime}=\boldsymbol{h}(t)
$$

has $N(\psi)$ linearly independent solutions $\boldsymbol{h}(t)$ in $\mathbf{2}^{2}$. To each of these, there corresponds a function

$$
f(t, \phi)=e^{\phi t-i \phi / 2+\psi t}\left(1+e^{2 \pi t}\right)^{-1} h(t)
$$

in $\mathfrak{\mathcal { Q }}^{2}$. This is an eigenvector of the operator $\boldsymbol{K}^{*}$ given by equation (5.17), which thus has at least $N(\psi)$ eigenvectors corresponding to the eigenvalue $1 / \bar{\gamma}$. Since $\boldsymbol{K}^{*}$ is the adjoint, on $\mathfrak{\mathscr { G }}^{2}$, of $\boldsymbol{K}$, it follows that $N \geqq N(\psi)$. Since it was found above that $N(\phi)$ $\geqq N$, we may conclude that $N=N(\phi)$. This means, in particular, that the set of eigenvectors of $K$ in $\mathfrak{S}^{2}$ exhausts all possibilities for eigenvectors of $K(\phi)$ in $\mathfrak{\Omega}^{2}$, and so each eigenvector of $K(\phi)$ must be the restriction to fixed $\phi$ of an eigenvector of $K$. This proves the theorem.

5.3. Applications. Consider equation (1.1), choose $g$ so that it is the boundary value of a function in $\mathfrak{S}^{2}$, and suppose that $\lambda$ is not real and positive. For any given function $V$, Theorem 3.5 provides a convenient criterion to determine whether the kernel of equation (1.1) is the boundary value $K(0)$ of a kernel $K$ in $\Re$. If the answer is yes, and 1 is not an eigenvalue of $K$, the equation can be solved for $f(r)$, next $f\left(r e^{i \phi}\right)$ can be found along the lines of Remark 2.11. Alternatively, one can first find $K$, next solve for $f\left(r e^{i \phi}\right)$. Either method yields the same result by Theorem 5.4.

Once $f(r)$ and $f\left(r e^{i \phi}\right)$ are known, these quantities can be inserted in the integrals (1.2) and (1.6). Since $K(0)$ depends on $\lambda$ in this case, so does the expression (1.2). Typically, it follows from the structure of the Fredholm solution for $f(r)$ that the integral (1.2) is a meromorphic function of $\lambda$ in the $\lambda$-plane cut along the positive real axis. For $\lambda \geqq 0$, however, the kernel of equation (1.1) presents difficulties, and so the expression (1.2) is no longer manageable.

Suppose now that $\lambda=l-i \varepsilon$, where $l \geqq 0$ and $\varepsilon \geqq 0$. Choose some fixed $\phi$, and consider the equation with kernel $K(\phi)$. Let this be solved for $f\left(r e^{i \phi}\right)$, and insert the solution in the integral (1.6). This gives the same result as the integral (1.2), by Corollary 2.8. Now examine the expression (1.6) as a function of $\lambda$. In typical cases, it is found from the Fredholm solution that (1.6) is a meromorphic function of $\lambda$ in the $\lambda$-plane cut along the line $\arg \lambda=\phi$. Thus, transferring from the integral (1.2) to (1.6) makes it possible to continue analytically in $\lambda$, the result being a function with a branch point at $\lambda=0$. Applications of this technique will be given in a separate paper. 


\section{Functions of several variables.}

6.1. Alternative characterization of the space $\Re$. There is another characterization of the space $\mathfrak{A}$, which throws new light on the ways in which $\mathfrak{\AA}$ can enter in applications. It is best understood if equation (3.10) is expressed in terms of new variables. Writing

$$
\begin{aligned}
r=\rho \cos \theta, \quad r^{\prime}=\rho \sin \theta & (0 \leqq \rho<\infty, 0 \leqq \theta \leqq \pi / 2), \\
t-t^{\prime}=u, \quad t+t^{\prime}=-2 v & (-\infty<u<\infty,-\infty<v<\infty), \\
K\left(r, r^{\prime}, \phi\right)=k(\rho, \phi, \theta), & K\left(t, t^{\prime}\right)=k(u, v)
\end{aligned}
$$

gives

$$
\begin{aligned}
k(\rho, \phi, \theta)=(2 \pi)^{-1} \int_{-\infty}^{\infty} \int_{-\infty}^{\infty} k(u, v)\left(\rho e^{i \phi}\right)^{-i u-1} & \\
& \times(\cos \theta \sin \theta)^{-i u / 2-1 / 2}(\tan \theta)^{-i v} d u d v
\end{aligned}
$$

Now perform the integration over $v$ and define

$$
k(u, \theta)=(2 \pi)^{-1 / 2}(\cos \theta \sin \theta)^{-i u / 2-1 / 2} \int_{-\infty}^{\infty} k(u, v)(\tan \theta)^{-i v} d v
$$

Well-known formulas for Mellin transforms show that

$$
\int_{0}^{\pi / 2} k(u, \theta) \bar{l}(u, \theta) d \theta=\int_{-\infty}^{\infty} \boldsymbol{k}(u, v) \bar{l}(u, v) d v
$$

for almost every $\boldsymbol{u}$ and every pair of functions $\boldsymbol{k}$ and $\boldsymbol{l}$ corresponding to functions $\boldsymbol{K}$ and $L$ in $\$$. Owing to equation (3.4), the integral

$$
\int_{-\infty}^{\infty}\left(1+e^{2 \pi u}\right)|k(u, \theta)|^{2} d u
$$

exists for almost every $\theta$ in $0 \leqq \theta \leqq \pi / 2$ and is an integrable function of $\theta$.

Now observe that

$$
k(\rho, \phi, \theta)=(2 \pi)^{-1 / 2} \int_{-\infty}^{\infty} k(u, \theta)\left(\rho e^{i \phi}\right)^{-i u-1} d u .
$$

This shows that $k(\rho, \phi, \theta)$ depends only on $\rho e^{i \phi}$ and $\theta$ and thereby leads to a number of observations.

REMARK 6.1. If $K\left(r, r^{\prime}, \phi\right)$ is a function in $\AA$, it depends only on $r e^{i \phi}$ and $r^{\prime} e^{i \phi}$.

THEOREM 6.2. Let $K\left(r, r^{\prime}, \phi\right)$ be a function in $\Re$ and let $k(\rho, \phi, \theta)$ be defined by equations (6.1) and (6.2). Then $k(\rho, \phi, \theta)$ depends only on $\rho e^{i \phi}$ and $\theta$. For almost every $\theta$ in $0 \leqq \theta \leqq \pi / 2$, the quantity

$$
\left(\rho e^{i \phi}\right)^{1 / 2} k(\rho, \phi, \theta)
$$

is an analytic function of $\rho e^{i \phi}$ which belongs to $\mathfrak{S}^{2}$. 
Proof. This follows with Theorem 2.9.

A corollary can be formulated with the help of the following definition.

Definition 6.3. The class of functions $f\left(\rho e^{i \phi}, \theta\right)$ which are defined in the region $0<\rho<\infty, 0<\phi<\pi, 0 \leqq \theta \leqq \pi / 2$, are regular analytic in $\rho e^{i \phi}$ for almost every $\theta$, and have the property that

$$
\int_{0}^{\pi / 2} d \theta \int_{0}^{\infty}\left|f\left(\rho e^{i \phi}, \theta\right)\right|^{2} \rho d \rho
$$

exists and is bounded, uniformly in $\phi$ for $0<\phi<\pi$, is denoted by $\mathfrak{S}^{2}(2)$.

In the above notation, the argument 2 refers to the functions in question depending on two variables, one complex and one real. Given a function $f$ in $\mathfrak{S}^{2}(2)$, there are boundary functions $f(\rho, \theta)$ and $f\left(\rho e^{i \pi}, \theta\right)$. This can be shown as in Lemmas 2.3 or 3.6. With the inner product

$$
(f, g)=\int_{0}^{\pi / 2} d \theta \int_{0}^{\infty}\left[f(\rho, \theta) \bar{g}(\rho, \theta)+f\left(\rho e^{i \pi}, \theta\right) \bar{g}\left(\rho e^{i \pi}, \theta\right)\right] \rho d \rho,
$$

the function class $\mathfrak{S}^{2}(2)$ becomes a Hilbert space. This is also denoted by $\mathfrak{S g}^{2}(2)$.

COROllaRY 6.4. If the function $K\left(r, r^{\prime}, \phi\right)$ is in $\AA$, then the function $k(\rho, \phi, \theta)$ defined according to equations (6.1) and (6.2) is in $\mathfrak{S}^{2}(2)$.

6.2. Generalizations. In much the same way as there is a class of operators $\AA$ on the Hilbert space $\mathfrak{S}^{2}$, there is a class of operators $\Re(2)$ on the space $\mathfrak{S}^{2}(2)$. Operators in $\Re(2)$ have kernels $K\left(\rho, \theta, \rho^{\prime}, \theta^{\prime}, \phi\right)$ such that

$$
\int_{0}^{\pi / 2} \int_{0}^{\pi / 2} d \theta d \theta^{\prime} \int_{0}^{\infty} \int_{0}^{\infty}\left|K\left(\rho, \theta, \rho^{\prime}, \theta^{\prime}, \phi\right)\right|^{2} \rho d \rho \rho^{\prime} d \rho^{\prime}
$$

exists and is bounded, uniformly in $\phi$ for $0<\phi<\pi$. The functions in question depend only on $\rho e^{i \phi}, \rho^{\prime} e^{i \phi}, \theta, \theta^{\prime}$ and are related to the elements of a Hilbert space $\mathfrak{S}^{2}(4)$ of functions of four variables, one complex and three real.

An obvious change of variables permits $\mathfrak{S}^{2}(2)$ to be regarded as a space of functions $f\left(r_{1} e^{i \phi}, r_{2} e^{i \phi}\right)$. Correspondingly, $\AA(2)$ is a space of operators having kernels $K\left(r_{1}, r_{2}, r_{1}^{\prime}, r_{2}^{\prime}, \phi\right)$. Theorem 3.5 can now be generalized to the statement that a function $K\left(r_{1}, r_{2}, r_{1}^{\prime}, r_{2}^{\prime}, \phi\right)$, defined on $0<r_{j}<\infty, 0<r_{j}^{\prime}<\infty \quad(j=1,2)$, $0<\phi<\pi$, is the kernel of an operator in $\Re(2)$ if and only if it is of the form

$$
\begin{aligned}
K\left(r_{1}, r_{2}, r_{1}^{\prime}, r_{2}^{\prime}, \phi\right)=(2 \pi)^{-2} \int_{-\infty}^{\infty} & K\left(t_{1}, t_{2}, t_{1}^{\prime}, t_{2}^{\prime}\right)\left(r_{1} e^{i \phi}\right)^{-i t_{1}-1 / 2}\left(r_{2} e^{i \phi}\right)^{-i t_{2}-1 / 2} \\
& \times\left(r_{1}^{\prime} e^{i \phi}\right)^{i t_{1}^{\prime}-1 / 2}\left(r_{2}^{\prime} e^{i \phi}\right)^{i t_{2}^{\prime}-1 / 2} d t_{1} d t_{2} d t_{1}^{\prime} d t_{2}^{\prime},
\end{aligned}
$$

with some function $\boldsymbol{K}$ satisfying

$$
\int_{-\infty}^{\infty}\left[1+\exp 2 \pi\left(t_{1}+t_{2}-t_{1}^{\prime}-t_{2}^{\prime}\right)\right]\left|K\left(t_{1}, t_{2}, t_{1}^{\prime}, t_{2}^{\prime}\right)\right|^{2} d t_{1} d t_{2} d t_{1}^{\prime} d t_{2}^{\prime}<\infty
$$


A Hilbert space $\mathfrak{S}^{2}(3)$, and, in fact, Hilbert spaces $\mathfrak{S}^{2}(n)$ of functions of $n$ variables $(n=2,3, \ldots)$, one complex and $n-1$ real, can be obtained by starting from functions $f\left(u, \theta_{1}, \ldots, \theta_{n-1}\right)$ satisfying

$$
\int_{0}^{\pi / 2} d \theta_{1} \cdots d \theta_{n-1} \int_{-\infty}^{\infty}\left(1+e^{2 \pi u}\right)\left|f\left(u, \theta_{1}, \ldots, \theta_{n-1}\right)\right|^{2} d u<\infty
$$

as in the expression (6.6), and taking inverse Mellin transforms with respect to $u$. On each space $\mathfrak{S E}^{2}(n)$, there is an operator class $\Re(n)$ consisting of integral operators whose kernels constitute a space $\mathfrak{S}^{2}(2 n)$.

6.3. Applications. The space $\mathfrak{S}^{2}(2)$ and the operator class $\mathfrak{\Re}(2)$ provide a convenient framework to study integral equations of the form

$$
\begin{aligned}
f\left(r_{1}, r_{2}\right)= & g\left(r_{1}, r_{2}\right) \\
& +\int_{0}^{\infty} \int_{0}^{\infty}\left[\left(r_{1}^{2}+r_{2}^{2}\right)^{1 / 2}-\lambda\right]^{-1} W\left(r_{1}, r_{2}, r_{1}^{\prime}, r_{2}^{\prime} ; \lambda\right) f\left(r_{1}^{\prime}, r_{2}^{\prime}\right) d r_{1}^{\prime} d r_{2}^{\prime} .
\end{aligned}
$$

Such equations arise when one investigates scattering in a system of three particles. Typically, $r_{1}$ and $r_{2}$ would be the kinetic energies of particle 2 relative to particle 1 , and particle 3 relative to the center of mass of particles 1 and 2 .

If $g$ in equation (6.15) is the boundary value of a function in $\mathfrak{S g}^{2}(2)$, and $W$ is such as to make the kernel of equation (6.15) the boundary value of a kernel in $\Re(2)$, then the ideas of $\S 5.3$ can be carried over to the present situation without difficulty. To investigate systems of $n$ particles, the appropriate generalization is to consider the space $\mathfrak{S}^{2}(n-1)$ and the operator class $\mathfrak{K}(n-1)$.

In applications to physics, the state of an $n$-particle system is described by a function in $\mathfrak{S}^{2}(n-1)$, an $n$-particle observable is represented by an operator mapping $\mathfrak{S}^{2}(n-1)$ into $\mathfrak{S}^{2}(n-1)$. Now let $A$ represent a two-particle observable. This means that it is an operator on $\mathfrak{S}^{2}$ into $\mathfrak{S}^{2}$. One would expect that it remains an observable if a third particle is added to the two-particle system. Thus, one would expect that $A$ is also an operator mapping $\mathfrak{S}^{2}(2)$ into $\mathfrak{S}^{2}(2)$. But $\mathfrak{S}^{2}(2)$ is essentially the same as the function space $\Re$, which in turn is the same as the operator space $\mathfrak{R}$. Hence, if there is to be an algebra $\mathfrak{A}$ of physically meaningful operators $A$ on $\mathfrak{S}^{2}$, one would wish $\mathfrak{R}$ to be an ideal in $\mathfrak{A}$. This is precisely the requirement met by the algebra $\mathfrak{A}$ discussed in $\$ 4$.

There is an obvious way to let operators in $\mathfrak{A}$ act on any space $\mathfrak{S}^{2}(n-1)$. In each case, it is found that $\mathfrak{I}(n-1)$ is an ideal in $\mathfrak{A}$. As implied by the notation, $\mathfrak{A}$ consists of two-particle operators. On $\mathfrak{S}^{2}(n-1)$, there are also algebras $\mathfrak{A}(m-1)$ of $m$-particle operators $(m=3, \ldots, n)$, the class $\mathfrak{R}(n-1)$ being an ideal in each $\mathfrak{A}(m-1)$.

Operators $A$ in $\mathfrak{A}(m-1)$ are associated with boundary values as $\phi$ tends to 0 . The boundary values can be extended to operators $A(0)$ on all of $\mathfrak{L}^{2}(n-1)$, as in Lemma 4.9. The set of all operators $A(0)$ obtained in this way is dense, in the strong topology on $\mathfrak{Q}^{2}(n-1)$, in the set of all bounded linear $m$-particle operators on 
$\mathfrak{R}^{2}(n-1)$, as in Theorem 4.22. Similarly, functions in $\mathfrak{S}^{2}(n-1)$ tend to boundary values as $\phi$ tends to 0 , the set of all boundary values being dense in $\mathfrak{Q}^{2}(n-1)$, as in Corollary 2.18. These two facts enable the conventional quantum mechanics of $n$-particle systems to be translated into a formalism on $\mathfrak{S}^{2}(n-1)$. The results of the present paper then enable certain problems in scattering theory to be tackled in a satisfactory way. The main idea is outlined in $\$ 1$. Details will be presented in a separate paper.

\section{REFERENCES}

1. E. Hille, Analytic function theory. Vol. 2, Introduction to Higher Math., Ginn, Boston, Mass., 1962. MR 34 \#1490.

2. K. Hoffman, Banach spaces of analytic functions, Prentice-Hall Series in Modern Analysis, Prentice-Hall, Englewood Cliffs, N. J., 1962. MR 24 \#A2844.

3. P. M. Morse and H. Feshbach, Methods of theoretical physics. Vol. 2, McGraw-Hill, New York, 1953. MR 15, 583.

4. R. G. Newton, Scattering theory of waves and particles, McGraw-Hill, New York, 1966. MR 36 \#4875.

5. R. E. A. C. Paley and N. Wiener, Fourier transforms in the complex domain, Amer. Math. Soc. Colloq. Publ., vol. 19, Amer. Math. Soc., Providence, R. I., 1934.

6. R. Schatten, Norm ideals of completely continuous operators, Ergebnisse der Mathematik und ihrer Grenzgebiete, N. F., Heft 27, Springer, Berlin, 1960. MR 22 \#9878.

7. L. I. Schiff, Quantum mechanics, 3rd ed., McGraw-Hill, New York, 1968.

8. F. Smithies, Integral equations, Cambridge Tracts in Math. and Math. Phys., No. 49, Cambridge Univ. Press, Cambridge, 1958. MR 21 \#3738.

9. E. C. Titchmarsh, Introduction to the theory of Fourier integrals, 2nd ed., Clarendon Press, Oxford, 1948.

10. C. van Winter, Theory of finite systems of particles. I. The Green function, Mat.-Fys. Skr. Danske Vid. Selsk. 2, no. 8 (1964). MR 34 \#1052.

11. - Theory of finite systems of particles. II. Scattering theory, Mat.-Fys. Skr. Danske Vid. Selsk. 2, no. 10 (1965). MR 38 \#909.

12. —_ "The $n$-body problem on a Hilbert space of analytic functions," in R. P. Gilbert and R. G. Newton, Analytic methods in mathematical physics, Gordon and Breach, London, 1970, pp. 569-578.

13. A. Zygmund, Trigonometrical series. Vol. 2, 2nd rev. ed., Cambridge Univ. Press, New York, 1959. MR 21 \#6498.

UNIVERSITY OF KeNTUCKy, LEXINGTON, KeNTUCKY 40506 